\title{
Mass detection in nanobeams from bending resonant frequency shifts
}

\author{
M. Dilena ${ }^{\mathrm{a}}$, M. Fedele Dell'Oste ${ }^{\mathrm{a}, *}$, J. Fernández-Sáez ${ }^{\mathrm{b}}$, A. Morassi ${ }^{\mathrm{a}}$, R. Zaera ${ }^{\mathrm{b}}$ \\ ${ }^{a}$ Polytechnic Department of Engineering and Architecture, University of Udine, via \\ Cotonificio 114, 33100 Udine, Italy \\ ${ }^{b}$ Department of Continuum Mechanics and Structural Analysis, Universidad Carlos III de \\ Madrid, Av. de la Universidad 30, 28911 Leganés, Madrid, Spain
}

\begin{abstract}
Nanobeams are frequently used as vibration based-sensors to detect mass changes caused, for instance, by attachment of foreign atoms/molecules or chemical/molecular absorption. This paper deals with the bending vibration of a uniform nanobeam carrying a single point mass (direct problem) as well as the identification of the attached mass (inverse problem). The nanobeam is described using the modified strain energy theory adapted to the Euler-Bernoulli beam model, and the natural vibration frequencies have been obtained. Under the assumption of small intensity of the concentrated mass, a solution of the inverse problem based on the measurement of the mass-induced shifts in the first two eigenfrequencies is proposed. Both the cases of simply supported and cantilever end conditions are discussed in detail. The theoretical method is verified by numerical simulation and numerical tests agree well with analytical results.

Keywords: Strain gradient theory, nanobeams, nanosensors, bending vibration, mass identification, inverse problems.
\end{abstract}

${ }^{*}$ Corresponding author. E-mail address: fedeledelloste.marta@spes.uniud.it 


\section{Introduction}

The use of nanostructures (carbon nanotubes, $C N T s$, graphene sheets, GSs, nanobeams and nanowires) as nano-sensors has focused a great interest of the scientific community over the past few years, and a wide range of applications

5 such as gas detection, early disease detection, gene mutation detection, deoxyribonucleic acid (DNA) sequencing have been devised. Several reviews and books have been recently published showing the different capabilities of the nanostructures as efficient nanosensors $[1,2,3,4,5]$.

The study of nanobeams-based sensors constitutes a very active research field (see $[6,4]$ and also Chapter 3 of [5], among others), due to the high sensitivity, low cost and fast response. Moreover, the high specificity of this kind of sensors makes them very suitable in many applications for detection purposes in liquid, gaseous, or vacuum media.

In this research we are interested in a class of mechanical nanoresonator sensors which could be modelled as Euler-Bernoulli beams and, in particular, in the use of vibration based-methods as identification techniques. The sensing principle is based on the measurement of the variations of lower order resonant frequencies caused by (unknown) additional masses located on certain positions of the initial system. The perturbation (added masses) can be caused by attachments of foreign atoms, molecules or virus particles on the surface of the nanobeams, as well as by chemical/molecular absorption or by the proteinprotein and protein-DNA interactions.

The conventional detection principle considers the mass perturbation as local addition of mass modelled, for detection purposes, as Dirac-delta point masses, having unknown intensities and locations, superimposed to the given mass density of the nanoresonator. This detection approach has been successfully used by Morassi and Dilena [7] to identify a point mass located on full-scale classical rods or beams (see also [8] for the case of rods), assuming that the added mass 
is small with respect to the total mass of the structure. The identification problem for a point mass located on a classical rod or beam, without any a priori assumption on the smallness of the attached mass, has been recently solved by Morassi and coworkers $[9,10]$. It is possible to show that this inverse problem occurs as an auxiliary problem in the identification of a (not necessarily small) crack in a rod or beam from resonant frequency data.

It is worth to point out that all the above cited works $[7,8,9,10]$ consider that the mechanical systems obey the laws of classical elasticity. However, it is well-known that classical continuum mechanics, due to its scale-free character, cannot predict the relevant size effects present in the mechanical behaviour of nanostructures which compose the nanoresonators. Therefore, other formula40 tions based on generalized continuum mechanics approaches taking into account this size-dependent behaviour must be explored.

Among the generalized continuum theories, we cite here three main groups:

1. The microcontinuum theory [11] including micropolar, microstretch and micromorphic $(3 M)$ theories (Cosserat micropolar elasticity [12] should be considered in this category, being the simplest formulation among $3 M$ theories).

2. The strain gradient elasticity theory, including the couple stress theory $[13,14,15]$, the first and second strain gradient theories of Mindlin [16, 17], the modified couple stress theory [18], and the modified strain gradient theory [19].

3. The nonlocal continuum mechanics theories initiated by Kröner [20], Krumhansl [21] and Kunin [22], simplified subsequently by Eringen and coworkers $[23,24,25]$, and formulated originally in integral form for linear homogeneous isotropic nonlocal elastic materials. 
nonlocal integral constitutive equation can be transformed into a differential form. Exploiting this transformation, which fairly simplify the analysis, and from the pioneering work by Peddieson et al. [27], the differential approach has been widely used to analyze the mechanical behaviour of nanostructures.

${ }_{60}$ The list of papers related with these applications is extremely long, and it is not feasible to quoted here the whole of them. Therefore, we refer to the recent published reviews on the application of nonlocal continuum theories to nanostructures $[28,29,30]$.

Several scholars used the Eringen elasticity theory to asses the vibrational ${ }_{65}$ behaviour of beams with attached masses [31, 32, 33, 34], and some attempts have been done to identify the added mass [32,33], but the analysed configurations are rather specific and a general formulation of the identification problem is still not available, to the authors's knowledge.

Nevertheless, the main drawback using the fully nonlocal elasticity theory of Eringen has been pointed out by Romano et al. [35], who shown that, in the majorities of the cases, the fully nonlocal elasticity theory leads to severely ill-posed problems that have no solution in general. The analysis developed in [35] clearly explains the paradoxical results found by several authors when this theory is applied to the static behaviour of nanobeams in tension [36] and also to the bending behaviour of nanocantilevers, both in static $[27,37,38,39,40]$ and in dynamic regime [41]. However, it can be shown that using the two-phase local/nonlocal constitutive model originally proposed by Eringen $[24,42]$, the ill-posedness of the purely nonlocal problem can be removed, see, for example, [35]. In this respect, several papers have been recently published using the so two-phase theory to study static bending [43, 44] and buckling [45] of EulerBernoulli nanobeams. The bending vibration of Euler-Bernoulli nanobeams has been also studied using the Finite Element $(F E)$ approach [46]. We refer to the recent paper by Fernández-Sáez and Zaera [47] for an analytical study of the free axial and bending vibration of a uniform beam modelled within the 
two-phase nonlocal elasticity theory.

Other non-classical elasticity theories arise as attractive alternatives to overcome the difficulties associated with the fully nonlocal elasticity framework. Thus, the modified couple stress theories [18] improving the classical couple stress formulations $[13,14,15]$, or the modified strain gradient elasticity proposed by Lam et al. [19] based on previous developments by Mindlin [17] and Fleck and Hutchinson [48]. These approaches need new additional equilibrium equations to govern the behavior of higher-order stresses, and the corresponding models contain new non-classical constants (one in the case of modified couple stress model, and three when the modified strain gradient is used) in addition to the two classical for isotropic linear elastic materials.

It is worth to note that the modified strain gradient formulation is more general that the couple stress theory. In fact, this last theory can be considered a special case of the proposed one by Lam et al. [19]. The classical continuum theory can be also recovered cancelling the scale parameters present in the strain gradient theory. Moreover, the results obtained with this theory are in good agreement with the experimental results corresponding to bending tests of micro-cantilevers [49]. Therefore, in this paper we use the modified strain theory to take into account size effects in nanobeams.

Regarding the use of this theory to model the mechanical behaviour of nanobeams, we mention the work by Kong et al. [50], who studied the static and dynamic bending behaviour of Euler-Bernoulli beams, and the research developed by Wang et al. [51], dealing with the analogous problem for Timoshenko beams. From this, the study of beams using the modified strain gradient model has led to numerous works. Akgoz and Civalek [52] derived analytical solutions for the buckling problem of axially loaded nano-sized beams with both uniform and variable cross section, using the Euler-Bernoulli theory. Later on, the same authors used a non-classical sinusoidal shear deformation to study buckling of a beam [53], and bending of beam embedded in an elastic medium [54]. 
Mohammadi and Mahzoon [55] investigated thermal effects on postbuckling of

the inverse problem in which the added mass is small with respect to the total mass of the nanobeam.

We shall study in detail the effect of the point mass intensity, location as well as the value of the scale parameter when the nanobeam is modelled using jected to simply supported or cantilever boundary conditions. For the case of small intensity of the concentrated mass, a first-order perturbative approach is 
used to estimate the natural frequencies of the nanobeam, and the approximate results are compared with those corresponding to the exact solution. Basing on the explicit expression of the first-order eigenfrequency change induced by the point mass, we are able to formulate and solve the inverse problem, consisting in the identification of the location and intensity of the point mass in a uniform nanobeam from minimal eigenfrequency data. In particular, for simply supported nanobeams, the method gives closed-form expressions of both the location and the intensity of the point mass in terms of a suitable pair of eigenfrequencies. To check the robustness of the identification method, the effect of frequency measurement errors on the estimated variables (mass intensity and location) has been illustrated by means of statistical analysis.

The paper is organized as follows. The problem of the free bending vibration of the nanobeam with and without point mass is briefly recalled in Section 2 . Section 3 is devoted to address the inverse problem of identifying the position and the intensity of the small point mass from eigenfrequency shifts. Applications and results of numerical simulations, both for the direct and the inverse eigenvalue problem, are reported and discussed in Section 4.

\section{Free bending vibration of a nanobeam carrying a point mass}

\subsection{Brief resume of the strain gradient theory for nanobeams in bending}

The modified strain gradient theory was presented by Lam et al. [19]. Besides the classical stress and strain definitions, higher-order stress and strain gradients are included in this formulation. Accordingly, additional equilibrium equations are needed and, for isotropic materials, these equations contain three non-classical material parameters in addition to the conventional Lame moduli. Brief resumes of the theory are presented, among other papers, in references [50], [59], [58].

Let us specialize the general modified strain gradient theory to the free undamped small motions of a slender straight uniform nanobeam of length $L$, 
vibrating transversally with respect to its longitudinal axis $x$. Assuming the kinematic hypotheses of the Euler-Bernoulli beams, the equation governing the transverse displacement $U(x, t)$ of the nanobeam reads as, see [50] for details,

$$
S U^{I V}(x, t)-K U^{V I}(x, t)=-\rho \ddot{U}(x, t),
$$

where $U^{\prime}(x, t)$ and $\dot{U}(x, t)$ indicate the first partial derivative of the function $U$ with respect to $x$ and $t$, respectively, $x \in(0, L)$ and $t>0$. The coefficient $\rho=\gamma A$ is the constant mass per unit length, where $\gamma$ is the volume mass density, and $A$ is the cross-sectional area. The constant coefficients $S$ and $K$ take the following expressions [59]:

$$
S=E I+2 G A l_{0}^{2}+\frac{120}{225} G A l_{1}^{2}+G A l_{2}^{2}, \quad K=I\left(2 G l_{0}^{2}+\frac{4}{5} G l_{1}^{2}\right) .
$$

It should be noted that the expression of the parameter $S$ differs from that given by Kong et al. [50]. For the case $l_{0}=l_{1}=l_{2}=l$, the above parameters take the form:

$$
S=E I+\frac{795}{225} G A l^{2}, \quad K=\frac{14}{5} I G l^{2} .
$$

In the above expressions, $I$ is the second moment of area about the axis through the centroid of the cross-section, at right angles to the plane of vibration. $G=$ $E /(2(1+\nu)), G>0$, is the shear modulus, defined in the classical way in terms of the Young's modulus $E, E>0$, and $\nu, \nu>0$, is the Poisson's ratio. The parameters $l_{0}, l_{1}$ and $l_{2}>0$, are the three additional materials constants needed to complete the model. Note that when $l_{0}=l_{1}=0$, the modified couple stress theory is recovered, while for $l_{0}=l_{1}=l_{2}=0$ this formulation coincides with the classical continuum one.

Using the separation of variables method, the transverse displacement $U(x, t)$ can be expressed as

$$
U(x, t)=u(x) \exp i \omega t,
$$

where $u=u(x)$ is the amplitude of the normal vibration mode (eigenfunction) associated to the natural (radian) frequency $\omega$, and $i=\sqrt{-1}$ is the imaginary 
unit. Substituting Eq.(4) into Eq.(1), the following ordinary differential equation is obtained

$$
S u^{I V}-K^{V I}=\lambda \rho u, \quad \text { in }(0, L),
$$

$\lambda=\omega^{2}$ being the eigenvalue. We shall be concerned with the following sets of classical and non-classical boundary conditions, see Kong et al. [50].

\section{Simply-Supported (S-S)}

Classical Boundary conditions:

$$
\begin{gathered}
u(0)=0, \quad-S u^{\prime \prime}(0)+K u^{I V}(0)=0, \\
u(L)=0, \quad-S u^{\prime \prime}(L)+K u^{I V}(L)=0 .
\end{gathered}
$$

Non-classical boundary conditions:

$$
u^{\prime \prime}(0)=0, \quad u^{\prime \prime}(L)=0 .
$$

\section{Cantilever (C-F)}

Classical Boundary conditions:

$$
\begin{aligned}
u(0) & =0, \quad u^{\prime}(0)=0, \\
-S u^{\prime \prime}(L)+K u^{I V}(L) & =0, \quad-S u^{\prime \prime \prime}(L)+K u^{V}(L)=0 .
\end{aligned}
$$

Non-classical boundary conditions:

$$
u^{\prime \prime}(0)=0, \quad K u^{\prime \prime \prime}(L)=0 .
$$

The non-classical boundary conditions selected above are only one of the two possible non-classical boundary conditions for nanobeam models based on strain gradient theories, see, for example, [49, 60, 61]. However, it should be noted that our identification method of the point mass is essentially based on the explicit expression of the sensitivity of the eigenvalues of the problem to the 
added mass. As will be shown in Section 3 (Theorem 3.1), this expression is independent of the boundary conditions of the eigenvalue problem and, therefore,

In this section we shall describe the free undamped bending vibration of a uniform nanobeam, of length $L$, carrying a point mass $M>0$ at $x=s, 0<s<$ $L$. To fix ideas, we assume that the nanobeam is supported at the ends, namely the transverse displacement and the classical bending moment vanish at the boundary, together with the strain gradient (non classical boundary condition). Therefore, the set of admissible configurations of the supported nanobeam is given by

$$
\begin{array}{r}
\mathcal{H}=\left\{f:(0, L) \rightarrow \mathbb{R} \mid f \in H^{3}(0, s) \cup H^{3}(s, L), f=f^{\prime \prime}=0 \text { at } x=0 \text { at } x=L,\right. \\
\left.[[f(s)]]=\left[\left[f^{\prime}(s)\right]\right]=\left[\left[f^{\prime \prime}(s)\right]\right]=0\right\}, \quad(12)
\end{array}
$$

where $[[f(s)]] \equiv\left(f\left(s^{+}\right)-f\left(s^{-}\right)\right)=\lim _{x \rightarrow s^{+}} f(x)-\lim _{x \rightarrow s^{-}} f(x)$, and $(\cdot)^{\prime}$ denotes the derivative of $(\cdot)$ with respect to $x$. Hereinafter, for any integer $m \geq 0$ and for any real numbers $a, b$, with $-\infty<a<b<+\infty, H^{m}(a, b)$ denotes the realvalued Hilbert space of the Lebesgue measurable functions $f:(a, b) \rightarrow \mathbb{R}$ such that $\int_{a}^{b}\left(f^{2}+\sum_{i=1}^{m}\left(\frac{d^{i} f}{d x^{2}}\right)^{2}\right)<+\infty$, where $\frac{d^{i} f}{d x^{2}}$ is the $i$ th weak derivative of $f$. Moreover, for $i=0, \ldots, m-1, \frac{d^{i} f}{d x^{i}}\left(a^{+}\right)$and $\frac{d^{i} f}{d x^{i}}\left(b^{-}\right)$are the traces of the function $f$ and its derivatives up to the order $m-1$ at $x=a$ and $x=b$, respectively. We recall that, when $m=0, H^{0}(a, b)$ coincides with the space $L^{2}(a, b)$ of the square integrable functions in $(a, b)$. 
Following Kong et al. [50], the Rayleigh's quotient $R: \mathcal{H} \backslash\{0\} \rightarrow \mathbb{R}$ of the nanobeam is

$$
R[\varphi]=\frac{\int_{0}^{L}\left(S\left(\varphi^{\prime \prime}\right)^{2}+K\left(\varphi^{\prime \prime \prime}\right)^{2}\right)}{M \varphi^{2}(s)+\int_{0}^{L} \rho \varphi^{2}},
$$
scopic properties of the system, as it was shown in (2).

Basing on the Variational Theory presented in [62], the eigenvalues can be defined by the following chain of minimum problems: for every $n \geq 1$, we have

$$
\widetilde{\lambda}_{n}=\min _{\varphi \in V_{n} \backslash\{0\}} R[\varphi]=R\left[\widetilde{u}_{n}\right]
$$

where

$$
V_{n}=\left\{g \in \mathcal{H} \mid M g(s) \widetilde{u}_{i}(s)+\int_{0}^{L} \rho g \widetilde{u}_{i}=0, i=1, \ldots, n-1\right\}
$$

and $\widetilde{\omega}_{n}=\sqrt{\widetilde{\lambda}_{n}}$ is the $n$th radian frequency of the free undamped vibration of the nanobeam and $\widetilde{u}_{n}=\widetilde{u}_{n}(x)$ is the corresponding eigenfunction.

The weak formulation of the eigenvalue problem (14)-(15) can be obtained by imposing the stationarity of the Rayleigh's quotient at $\varphi=\widetilde{u}$, where $\widetilde{u} \in$ $\mathcal{H} \backslash\{0\}$. Let $\delta>0$ be a given number. For every $\epsilon \in[-\delta, \delta]$ and for every $v \in \mathcal{H}$, the function

$$
\Phi:[-\delta, \delta] \rightarrow \mathbb{R}, \quad \Phi(\epsilon)=R[\widetilde{u}+\epsilon v],
$$

is continuous and with continuous first derivative in $[-\delta, \delta]$. The stationarity of 220 $R[\cdot]$ at $\widetilde{u}$ requires $\left.\Phi^{\prime}(\epsilon)\right|_{\epsilon=0}=0$. By imposing this condition and elaborating, we obtain the wished weak formulation of the eigenvalue problem (14)-(15):

to find $\{\widetilde{u} \in \mathcal{H} \backslash\{0\}, \widetilde{\lambda} \in \mathbb{R}\}$ such that

$$
\int_{0}^{L}\left(S \widetilde{u}^{\prime \prime} v^{\prime \prime}+K \widetilde{u}^{\prime \prime \prime} v^{\prime \prime \prime}\right)=\widetilde{\lambda}\left(M \widetilde{u}(s) v(s)+\int_{0}^{L} \rho \widetilde{u} v\right) \quad \text { for every } v \in \mathcal{H} .
$$

It should be noticed that, under our assumptions on the coefficients and on the functional space $\mathcal{H}$, there exists an infinite sequence of real positive eigenvalues $\left\{\widetilde{\lambda}_{n}\right\}_{n=1}^{\infty}$, with $0<\widetilde{\lambda}_{1}<\widetilde{\lambda}_{2}<\ldots$ and $\lim _{n \rightarrow \infty} \widetilde{\lambda}_{n}=\infty$, see [63]. 
Finally, the strong or differential formulation of the eigenvalue problem can be derived from the weak formulation by assuming enough additional regularity on $\widetilde{u}$. By integrating by parts in (17) in the subintervals $(0, s)$ and $(s, L)$, and following a standard procedure of Calculus of Variations, we have

$$
\begin{aligned}
& \left(-S \widetilde{u}^{\prime \prime}+K \widetilde{u}^{I V}\right)(0) v^{\prime}(0)+\left(S \widetilde{u}^{\prime \prime}-K \widetilde{u}^{I V}\right)(L) v^{\prime}(L)+ \\
& +\left[\left[\left(S \widetilde{u}^{\prime \prime \prime}-K \widetilde{u}^{V}\right)(s)\right]\right] v(s)+\left[\left[\left(-S \widetilde{u}^{\prime \prime}+K \widetilde{u}^{I V}\right)(s)\right]\right] v^{\prime}(s)+\left[\left[-K \widetilde{u}^{\prime \prime \prime}(s)\right]\right] v^{\prime \prime}(s)+ \\
& +\int_{0}^{L}\left(S \widetilde{u}^{I V}-K \widetilde{u}^{V I}\right) v=\widetilde{\lambda}\left(M \widetilde{u}(s) v(s)+\int_{0}^{L} \rho \widetilde{u} v\right), \quad(18)
\end{aligned}
$$

225 for every $v \in \mathcal{H}$. By the arbitrariness of $v \in \mathcal{H}$, the strong formulation of the eigenvalue problem consists in determining $\left\{\widetilde{u} \in\left(H^{6}(0, s) \cup H^{6}(s, L)\right) \backslash\{0\}\right.$, $\left.\tilde{\lambda} \in \mathbb{R}^{+}\right\}$such that

$$
\left\{\begin{array}{l}
S \widetilde{u}^{I V}-K \widetilde{u}^{V I}=\tilde{\lambda} \rho \widetilde{u}, \quad \text { in }(0, s) \cup(s, L), \\
\widetilde{u}(0)=\widetilde{u}^{\prime \prime}(0)=0 \\
\left(-S \widetilde{u}^{\prime \prime}+K \widetilde{u}^{I V}\right)(0)=0 \\
{[[\widetilde{u}(s)]]=0} \\
{\left[\left[\widetilde{u}^{\prime}(s)\right]\right]=0} \\
{\left[\left[\widetilde{u}^{\prime \prime}(s)\right]\right]=0} \\
{\left[\left[\left(-S \widetilde{u}^{\prime \prime \prime}+K \widetilde{u}^{V}\right)(s)\right]\right]=-\widetilde{\lambda} M \widetilde{u}(s),} \\
{\left[\left[\left(-S \widetilde{u}^{\prime \prime}+K \widetilde{u}^{I V}\right)(s)\right]\right]=0} \\
{\left[\left[K \widetilde{u}^{\prime \prime \prime}(s)\right]\right]=0} \\
\left(-S \widetilde{u}^{\prime \prime}+K \widetilde{u}^{I V}\right)(L)=0 \\
\widetilde{u}(L)=\widetilde{u}^{\prime \prime}(L)=0
\end{array}\right.
$$

The unperturbed eigenvalue problem can be deduced from (19)-(29) by taking ${ }_{230} M=0$. It consists in finding $\left\{u \in H^{6}(0, L) \backslash\{0\}, \lambda \in \mathbb{R}^{+}\right\}$such that 


$$
\left\{\begin{array}{l}
S u^{I V}-K u^{V I}=\lambda \rho u, \quad \text { in }(0, L) \\
u(0)=u^{\prime \prime}(0)=u^{I V}(0)=0 \\
u(L)=u^{\prime \prime}(L)=u^{I V}(L)=0
\end{array}\right.
$$

see also Kong et al. [50] (Section 4).

The strong formulation of both the perturbed and unperturbed problems (19) $-(29)$ and (30)-(32), respectively, will be used in the next sections to find 235 closed form expressions for the eigenpairs of uniform nanobeams.

\section{Identification of a small point mass in a nanobeam by two resonant frequencies}

In this section we consider the problem of identifying a small point mass in a nanobeam by minimal resonant frequency data, that is we assume

$$
M<<\rho L
$$

We first study the first-order effects of the added mass on the eigenvalues of the unperturbed problem (Section 3.1). Next, we shall apply the resonant frequency shift formula (35) to identify the point mass in a nanobeam under supported (Section 3.2) and cantilever (Section 3.3) end conditions.

\subsection{First-order eigenvalue shift}

The first order approximation for the eigenvalues of the perturbed problem can be obtained by using the following result.

Theorem 3.1. Denoting by $\left(\widetilde{\lambda}_{n}, \widetilde{u}_{n}\right)$ the $n$th eigenpair of (19)-(29), $n \geq 1$, for

a given position $s \in(0, L)$ of the point mass, the function $\widetilde{\lambda}_{n}=\widetilde{\lambda}_{n}(M)$ is a $C^{1}$-function in $(0, \infty)$, and we have

$$
\frac{\partial \widetilde{\lambda}_{n}}{\partial M}=-\widetilde{\lambda}_{n} \frac{\widetilde{u}_{n}^{2}(s)}{M \widetilde{u}_{n}^{2}(s)+\int_{0}^{L} \rho \widetilde{u}_{n}^{2}} .
$$


A proof of Theorem 3.1 and expression (34) can be obtained by adapting the arguments of the proof of Proposition 3.1 in [58] to the weak formulation (17). We omit the details.

By (34), the first order approximation of the $n$th perturbed eigenvalue is

$$
\widetilde{\lambda}_{n}(M)=\lambda_{n}-\lambda_{n} u_{n}^{2}(s) M
$$

where the mass-normalization condition $\int_{0}^{L} \rho u_{n}^{2}=1$ has been taken into account.

We conclude this section with two remarks. First, it should be noted that the expression (35) of the resonant frequency induced shift by the small point mass $M$ is independent from the boundary conditions. Second, it is easy to prove that expression (35) can be derived also for smooth variable (positive) coefficients $S=S(x), K=K(x)$ and $\rho=\rho(x)$.

\subsection{Identification in a supported nanobeam}

The eigenpairs of (30)-(32) for the unperturbed nanobeam under supported end conditions have the following closed-form expression:

$$
\begin{gathered}
\lambda_{n}=\left(\frac{n \pi}{L}\right)^{6}\left[\frac{K}{\rho}+\frac{S}{\rho} \frac{1}{\left(\frac{n \pi}{L}\right)^{2}}\right], \\
u_{n}(x)=\sqrt{\frac{2}{\rho L}} \sin \left(\frac{n \pi x}{L}\right), \quad n \geq 1 .
\end{gathered}
$$

In fact, a direct inspection shows that (36)-(37) are eigenpairs of (30)-(32). In order to prove that these are the all eigenpairs of (30)-(32), we proceed by contradiction. Let us assume there exists another eigenfunction $y, y \in H^{6}(0, L) \backslash$ $\{0\}$, associated to the eigenvalue $\lambda$, with $\lambda \neq \lambda_{n}$ for every $n \geq 1$. By the weak formulation of the eigenvalue problem for the eigenpairs $\left(u_{n}, \lambda_{n}\right)$ and $(y, \lambda)$, we have

$$
\int_{0}^{L}\left(S u_{n}^{\prime \prime} y^{\prime \prime}+K u_{n}^{\prime \prime \prime} y^{\prime \prime \prime}\right)=\lambda_{n} \int_{0}^{L} \rho u_{n} y
$$




$$
\int_{0}^{L}\left(S u_{n}^{\prime \prime} y^{\prime \prime}+K u_{n}^{\prime \prime \prime} y^{\prime \prime \prime}\right)=\lambda \int_{0}^{L} \rho u_{n} y
$$

and then, subtracting term by term and recalling that $\lambda \neq \lambda_{n}$, we have

$$
\int_{0}^{L} u_{n} y=0, \quad \text { for every } n \geq 1 .
$$

Equation (40) states that every Fourier's coefficient of the function $y$, evaluated on the family $\mathcal{F}=\left\{\sin \left(\frac{n \pi x}{L}\right)\right\}_{n=1}^{\infty}$ vanishes. Since the family $\mathcal{F}$ is a complete family in $L^{2}(0, L)$, we have $y=0$ in $[0, L]$, a contradiction.

By substituting the expressions (36)-(37) in (35) we obtain

$$
C_{n}^{S}=M \sin ^{2}\left(\frac{n \pi s}{L}\right)
$$

with

$$
C_{n}^{S}=-\frac{\left(\widetilde{\lambda}_{n}-\lambda_{n}\right)}{\lambda_{n}} \frac{\rho L}{2}, \quad n \geq 1 .
$$

Therefore, the procedure shown in Section 4 of [58] can be used to identify the point mass by the pair of natural frequencies $\left(\lambda_{n}, \lambda_{2 n}\right), n \geq 1$. More precisely, if $C_{n}^{S}>0$, then the following closed-form expressions for mass intensity and position hold

$$
\begin{gathered}
M=\frac{C_{n}^{S}}{1-\frac{C_{2 n}^{S}}{4 C_{n}^{S}}}, \\
P=\cos \left(\frac{2 n \pi s}{L}\right)=\frac{C_{2 n}^{S}}{2 C_{n}^{S}}-1 .
\end{gathered}
$$

Conversely, if $C_{n}^{S}=0$ for certain $n \geq 2$, then $P=1$ and the point mass 260 is located in one of the nodal points of the $n$th vibration mode. The mass intensity remains undetermined in this case. It should be noticed by (44) that the measurement of the first two natural frequencies determines uniquely the position of the point mass up to symmetry with respect to $x=\frac{L}{2}$. 


\subsection{Identification in a cantilever nanobeam}

265 The eigenpairs $\left\{u \in H^{6}(0, L) \backslash\{0\}, \lambda \in \mathbb{R}^{+}\right\}$of the unperturbed uniform nanobeam, with coefficients $\{S, K, L\}$ and under cantilever end conditions, are the solutions to the eigenvalue problem

$$
\left\{\begin{array}{l}
S u^{I V}-K u^{V I}=\omega^{2} \rho u, \quad \text { in }(0, L) \\
u(0)=u^{\prime}(0)=u^{\prime \prime}(0)=0 \\
-S u^{\prime \prime}(L)+K u^{I V}(L)=0 \\
-S u^{\prime \prime \prime}(L)+K u^{V}(L)=0 \\
K u^{\prime \prime \prime}(L)=0
\end{array}\right.
$$

see, for instance, [50], where $\omega=\sqrt{\lambda}, u=u(x)$ is the radian frequency and the amplitude of the transverse harmonic vibration, respectively. Unlike the simplysupported case discussed in the previous section, the eigenvalue problem (45)(49) does not admit closed-form expressions for the eigensolutions. Therefore, a non trivial solution to (45) is sought as $u(x)=\exp (\alpha x)$, where the exponent $\alpha, \alpha \in \mathbb{C}$ and $\alpha^{2}=z$, can be found by solving the polynomial equation

$$
f(z, \omega)=K z^{3}-S z^{2}+\omega^{2} \rho=0
$$

Depending on the value of $\omega^{2}$, we can distinguish the following three case:

i) $(0<) \omega^{2}<\frac{4}{27} \frac{S^{3}}{\rho K^{2}}$ (low frequency regime);

ii) $\omega^{2}>\frac{4}{27} \frac{S^{3}}{\rho K^{2}}$ (high frequency regime);

iii) $\omega_{*}^{2}=\frac{4}{27} \frac{S^{3}}{\rho K^{2}}$ (critical frequency value $\left.\omega_{*}\right)$.

In all cases cases i)-iii), there exists exactly one simple negative root of (50), say $z_{1}$, with $-\frac{S}{3 K}<z_{1}<0, z_{1}<-\frac{S}{3 K}$ for $\omega<\omega_{*}, \omega>\omega_{*}$, respectively. In addition, in case i) there exist two simple real positive roots of $f(z, \omega)$, say $z_{2}$ and $z_{3}$, with $0<z_{2}<\frac{2 S}{3 K}$ and $\frac{2 S}{3 K}<z_{3}<\frac{S}{K}$. Therefore, the general solution to 
the differential equation (45) can be written as

$$
\begin{aligned}
& u(x)=c_{1} \sin \left(\sqrt{-z_{1}} x\right)+c_{2} \cos \left(\sqrt{-z_{1}} x\right)+c_{3} \sinh \left(\sqrt{z_{2}} x\right)+ \\
& +c_{4} \cosh \left(\sqrt{z_{2}} x\right)+c_{5} \sinh \left(\sqrt{z_{3}} x\right)+c_{6} \cosh \left(\sqrt{z_{3}} x\right),
\end{aligned}
$$

where $c_{i}, i=1, \ldots, 6$, are real constants. In the high frequency regime ii), the equation (50) has the simple negative root $z_{1}$ and two complex conjugate roots $z_{2}, z_{3}=\bar{z}_{2}$, and the general solution to (45) takes the form

$$
\begin{aligned}
u(x) & =c_{1} \sin \left(\sqrt{-z_{1}} x\right)+c_{2} \cos \left(\sqrt{-z_{1}} x\right)+c_{3} \exp (a x) \sin (b x)+ \\
& +c_{4} \exp (-a x) \sin (b x)+c_{5} \exp (a x) \cos (b x)+c_{6} \exp (-a x) \cos (b x),
\end{aligned}
$$

where $c_{i}, i=1, \ldots, 6$, are real constants and $(a+i b)^{2}=z_{2}$. Finally, the general solution to the limit case iii) can be written as

$$
\begin{aligned}
& u(x)=c_{1} \sin \left(\sqrt{-z_{1}} x\right)+c_{2} \cos \left(\sqrt{-z_{1}} x\right)+ \\
& +\left(c_{3}+c_{4} x\right) \exp \left(-\sqrt{\frac{2 S}{3 K}} x\right)+\left(c_{5}+c_{6} x\right) \exp \left(\sqrt{\frac{2 S}{3 K}} x\right) .
\end{aligned}
$$

The boundary conditions (46)-(49) are written in terms of the general solution to obtain a $6 \times 6$ homogeneous linear system $\mathbf{M}\left(\omega^{2}\right) \mathbf{c}=\mathbf{0}$ in the unknown vector ${ }_{275} \mathbf{c}=\left(c_{1}, \cdots, c_{6}\right)$. In order to determine the natural frequencies as the roots of the frequency equation $\operatorname{det} \mathbf{M}\left(\omega^{2}\right)=0$, the following numerical procedure was used. Once an initial value for $\omega$ was set, say $\widetilde{\omega}$, the third order polynomial equation (50) was solved with respect to the variable $z$ and the expression of the general solution to (45) was determined. By imposing the six boundary conditions of the problem, the value of $\operatorname{det} \mathbf{M}\left(\widetilde{\omega}^{2}\right)$ was calculated. Next, by repeating the procedure for $\widetilde{\omega}+\Delta \omega$, where $\Delta \omega$ is a proper frequency resolution, the graph of the function $\operatorname{det} \mathbf{M}\left(\omega^{2}\right)$ was reconstructed in a given frequency interval. Eigenfrequencies are evaluated by a bisection method applied between two consecutive values of $\omega$ corresponding to a change of sign of $\operatorname{det} \mathbf{M}\left(\omega^{2}\right)$. ${ }_{285}$ Finally, for each eigenfrequency value, after solving $\mathbf{M}\left(\omega^{2}\right) \mathbf{c}=\mathbf{0}$, the vector $\mathbf{c}$ 
of the constants of integration is calculated and the corresponding normal mode is determined.

At this stage, the identification of the small point mass $M$ from the first two eigenvalue shifts $\delta \lambda_{1}, \delta \lambda_{2}$ can be formulated and solved. Writing the first-order eigenvalue expression (35) for $n=1,2$, and dividing side-by-side, the possible positions of the mass are the solutions of the equation

$$
\frac{C_{2}^{C}}{C_{1}^{C}}=\frac{u_{2}^{2}(s)}{u_{1}^{2}(s)} \equiv g(s)
$$

where

$$
C_{n}^{C}=-\frac{\left(\widetilde{\lambda}_{n}-\lambda_{n}\right)}{\lambda_{n}}
$$

$n=1,2$. A typical behaviour of the function $g=g(s)$ is plotted in Figure 1 for the nanobeam considered in Section 4. Postponing the general study of $g=g(s)$ to future work, in the sequel we shall investigate numerically on the solutions of (54). It should be noticed that the ratio $\delta \lambda_{2} / \delta \lambda_{1}$ allows the unique localization of the point mass whenever the ratio value is 'large' enough, e.g., greater than the critical value 1.00076 in Figure 1. On the contrary, two different positions correspond to the same value of $\delta \lambda_{2} / \delta \lambda_{1}$ when the frequency ratio is smaller than 1.00076. We refer to the second part of Section 4.3 for an application.

\subsection{An extension: identification of two point masses}

We conclude Section 3 by presenting an extension of the above results to multiple point mass detection. The analysis developed in the sequel will be focussed on the identification of two point masses $\left(s_{1}, M_{1}\right),\left(s_{2}, M_{2}\right)$ in a uniform supported nanobeam from minimal resonant frequency shift data.

Let us assume that $0<s_{1}<s_{2}<L$. The undamped free transverse vibrations of the perturbed nanobeam satisfy the boundary value problem (19)(29), where the differential equation (19) holds in the set $\left(0, s_{1}\right) \cup\left(s_{1}, s_{2}\right) \cup\left(s_{2}, L\right)$ and the jump conditions (22)-(27) hold at the cross-sections $x=s_{1}, x=s_{2}$. On 
proceeding as in Section 3.1 and with the above notation, the first order change of the $n$th eigenvalue is given by

$$
C_{n}^{S}=M_{1} \sin ^{2}\left(\frac{n \pi s_{1}}{L}\right)+M_{2} \sin ^{2}\left(\frac{n \pi s_{2}}{L}\right),
$$

where $C_{n}^{S}$ is defined in (42), $n \geq 1$.

We formulate the inverse problem in terms of the changes in the first four natural frequencies. By writing (56) for $n=1,2,3,4$, we obtain the following system of nonlinear equations to be solved with respect to the four parameters $\left(s_{1}, M_{1}\right),\left(s_{2}, M_{2}\right)$ :

$$
\left\{\begin{array}{l}
M_{1} \sin ^{2} \frac{\pi s_{1}}{L}+M_{2} \sin ^{2} \frac{\pi s_{2}}{L}=C_{1}^{S} \\
M_{1} \sin ^{2} \frac{2 \pi s_{1}}{L}+M_{2} \sin ^{2} \frac{2 \pi s_{2}}{L}=C_{2}^{S}, \\
M_{1} \sin ^{2} \frac{3 \pi s_{1}}{L}+M_{2} \sin ^{2} \frac{3 \pi s_{2}}{L}=C_{3}^{S} \\
M_{1} \sin ^{2} \frac{4 \pi s_{1}}{L}+M_{2} \sin ^{2} \frac{4 \pi s_{2}}{L}=C_{4}^{S}
\end{array}\right.
$$

where

$$
C_{i}^{S}>0, i=1,2,3, C_{4}^{S} \geq 0
$$

The unperturbed nanobeam is symmetric with respect to the mid-point of the beam axis. Therefore, the configurations $\left\{\left(s_{1}, M_{1}\right),\left(s_{2}, M_{2}\right)\right\},\left\{\left(L-s_{1}, M_{1}\right),(L-\right.$ $\left.\left.s_{2}, M_{2}\right)\right\},\left\{\left(L-s_{1}, M_{1}\right),\left(s_{2}, M_{2}\right)\right\},\left\{\left(s_{1}, M_{1}\right),\left(L-s_{2}, M_{2}\right)\right\}$ cannot be distinguished from natural frequency data. Taking into account this intrinsic nonuniqueness of the problem, it is not restrictive to assume

$$
0<s_{1}<s_{2} \leq \frac{L}{2}
$$

It is worth noticing that system (57)-(60) shows a structure similar to that of the system (13) - (16) encountered in [64] in the identification of two open cracks of different severity in a (classical) bending beam under simply supported end conditions. Therefore, we take advantage of the identification method illustrated in [64] for finding the explicit solution to the nonlinear system (57)-(60). Omitting the details and referring the interested reader to the above mentioned 
paper for precise statements, in the sequel we simply recall the main result: the knowledge of the first four natural frequencies allows to uniquely determine the intensity and the location of the two point masses, up to symmetry with respect to the mid-span cross-section. Remarkably, closed-form expressions both for the mass positions and intensities can be obtained in terms of the natural frequency data.

\section{Applications}

Aim of this section is three-fold. First, we shall evaluate the accuracy of the perturbation approach illustrated in Section 3.1 in estimating the first two natural frequencies of a nanobeam with a small point mass. Second, we shall apply the resonant-based detection method described in Section 3.2 and 3.3 to identify the position and the intensity of the point mass. For the sake of completeness, both the supported and cantilever end conditions are considered in this analysis. Finally, in the last part of the section we shall investigate on the stability of the identification method to errors on the input data.

\subsection{The specimen}

For illustration purposes, the material properties of the nanobeam used in the calculations are those used by Kong et al. [50], i.e., $E=1.44 \mathrm{GPa}$ and $\nu=0.38$. We also assume that the three material length scale parameters are equal, i.e., $l_{0}=l_{1}=l_{2}=l=17.6 \mu \mathrm{m}$, and that the equivalent cross-section is rectangular with $b / h=2$ and $I=\frac{b h^{3}}{12}$. The geometrical properties of the nanobeam are collected in Table 1.

\subsection{Exact versus perturbative eigensolutions}

The variation of the first two eigenvalues $\widetilde{\lambda}_{n}, n=1,2$, with respect to the mass intensity $M$ is plotted in Figures 2-3, and 4-5 for supported and cantilever end conditions, respectively, and for different values of $h$ such as 
$h / l=1,2,5,10$. The mass $M$ is normalized to the total mass $\rho L$ of the nanobeam, and three positions of the point mass are considered in simulations, namely $s / L=0.10,0.25,0.50$ and $s / L=0.10,0.50,0.90$ in the supported and cantilever case, respectively. Eigenvalues $\widetilde{\lambda}_{n}$ are normalized to the corresponding eigenvalues $\lambda_{0 n}$ of the "classical" local beam, that is the beam with $K=0$, $S=E I$, and without the attached mass. In particular, the figures compare the exact values of the eigenvalues, as determined by solving the problem (19)(29) for the supported case and its analogous for the cantilever case, and their approximate values obtained via the perturbative solution (35). As an example, and adopting the notation of Section 3.3, the frequency equation of the supported nanobeam in the low frequency regime (i.e., see case i) $\lambda<\omega_{*}^{2}$ ) is

$$
\begin{aligned}
& f^{S-S}(\lambda)=K \sqrt{-z_{1}} \sqrt{z_{2}} \sqrt{z_{3}}\left(z_{1}-z_{2}\right)^{2}\left(z_{1}-z_{3}\right)^{2}\left(z_{2}-z_{3}\right)\left(z_{3}-z_{2}\right) . \\
& \cdot \sin \left(L \sqrt{-z_{1}}\right) \sinh \left(L \sqrt{z_{2}}\right) \sinh \left(L \sqrt{z_{3}}\right)-\frac{1}{2} M\left(z_{1}-z_{2}\right)\left(z_{1}-z_{3}\right)\left(z_{2}-z_{3}\right) . \\
& \cdot\left\{\sqrt{-z_{1}} \sqrt{z_{3}} \lambda\left(z_{1}-z_{3}\right) \sin \left(L \sqrt{-z_{1}}\right) \sinh \left(L \sqrt{z_{2}}\right) \sinh \left(L \sqrt{z_{3}}\right) \sinh \left(2 s \sqrt{z_{2}}\right)-\right. \\
& -\sqrt{-z_{1}} \sqrt{z_{2}} \lambda\left(z_{1}-z_{2}\right) \sin \left(L \sqrt{-z_{1}}\right) \sinh \left(L \sqrt{z_{2}}\right) \sinh \left(L \sqrt{z_{3}}\right) \sinh \left(2 s \sqrt{z_{3}}\right)+ \\
& \quad+\sqrt{z_{2}} \sqrt{z_{3}} \lambda\left(z_{3}-z_{2}\right) \sinh \left(L \sqrt{z_{2}}\right) \sinh \left(L \sqrt{z_{3}}\right) \cos \left(\sqrt{-z_{1}}(L-2 s)\right)- \\
& -2 \sqrt{-z_{1}} \sqrt{z_{3}} \lambda\left(z_{1}-z_{3}\right) \sin \left(L \sqrt{-z_{1}}\right) \cosh \left(L \sqrt{z_{2}}\right) \sinh \left(L \sqrt{z_{3}}\right) \sinh ^{2}\left(s \sqrt{z_{2}}\right)+ \\
& +2 \sqrt{-z_{1}} \sqrt{z_{2}} \lambda\left(z_{1}-z_{2}\right) \sin \left(L \sqrt{-z_{1}}\right) \sinh \left(L \sqrt{z_{2}}\right) \cosh \left(L \sqrt{z_{3}}\right) \sinh ^{2}\left(s \sqrt{z_{3}}\right)- \\
& \left.\quad-\sqrt{z_{2}} \sqrt{z_{3}} \lambda\left(z_{3}-z_{2}\right) \cos \left(L \sqrt{-z_{1}}\right) \sinh \left(L \sqrt{z_{2}}\right) \sinh \left(L \sqrt{z_{3}}\right)\right\} .
\end{aligned}
$$

An expression analogous to (63) has been determined for the uniform nanocantilever with a single point mass. However, the closed-form expression is rather huge and it is not reported here for the sake of brevity.

Numerical results suggest that, besides on the mass intensity $M$, the agreement between exact and first-order eigensolutions depends on the position of the attached point mass. Typically, the smaller the amplitude $u_{n}(s)$, the better the accuracy. In the supported case, the maximum difference is encountered at $s / L=0.50$ and $s / L=0.25$ for the first and second mode, respectively. 
Maximum deviations are about 1, 4, $9 \%$ (first mode) and 1, 5, $11 \%$ (second

345

mode) for $M /(\rho L)=0.05,0.10,0.15$, respectively. In the cantilever case, percentage errors on the first eigenfrequency for $s / L=0.90$ are about $2,9,20 \%$ for $M /(\rho L)=0.05,0.10,0.15$, respectively. Numerical results show that the accuracy of the perturbative frequency estimate seems to be quite uniform with respect to the scale factor $l$, at least in the range of values considered.

\subsection{Identification results}

In this section, numerical applications of the identification method are presented, both for supported and cantilever end conditions. Resonant frequencies are obtained by solving exactly the direct problem in referential and perturbed configuration for different position and intensity of the point mass. Simulations are performed with noise-free data. It should be noticed, however, that even in these cases an intrinsic error is present on the eigenfrequency data, since the higher order terms on $M$ are neglected in the first order Taylor series approximation (35).

The first series of simulations refers to the supported nanobeam. Figure 6 shows the results varying continuously the position $s / L$ of the point mass within the interval $[0,1 / 2]$ and using selected values of the normalized mass intensity $M /(\rho L)=0.010,0.025,0.050,0.100,0.150,0.200$. These values correspond approximately to maximum relative shifts $\delta \lambda_{n} / \lambda_{n}$ equal to $2,5,9,17,23,29 \%$ and $2,5,9,16,22,26 \%$ for $n=1$ and $n=2$, respectively. Identification errors on the mass position are of order of few points per cent and, surprisingly enough, the estimate remains accurate even for high mass values, e.g., the maximum error is about $5 \%$ for $M /(\rho L)=0.200$. In particular, the discrepancy vanishes when the point mass approaches either the support or the mid-point of the nanobeam, and the maximum error is typically attained at $s / L \simeq 0.200$. The determination of the mass intensity is less accurate, with errors up to $15-30 \%$ and $40-50 \%$ for $M /(\rho L)=0.050-0.100$ and $M /(\rho L)=0.150-0.200$, respectively. More 
precisely, when the point mass is approaching the support, the estimate of the intensity becomes very inaccurate and the identification is seriously compromised. This behaviour is a consequence of the vanishing sensitivity of both the eigenvalues (e.g., $\left.u_{n}(0)=0, n=1,2\right)$ and of the occurrence of an indeterminate quotient $0 / 0$ in the expression (43) of $M$ as $s \rightarrow 0^{+}$.

Figure 7 shows the results of identification for the cantilever nanobeam. According with the analysis developed in Section 3.3 (see also Figure 1), the knowledge of the changes in the first two eigenfrequencies is not always sufficient for the unique localization of the point mass. The results presented in Figure 7 refer to the actual position of the point mass only. In particular, the mass intensity $\mathrm{M}$ has been evaluated via (35) with $n=1$. As in the supported case, mass location errors are almost negligible, whereas the accuracy on mass intensity evaluation is worse, with maximum errors up to $30 \%, 40 \%$ for $M /(\rho L)=0.150,0.200$, respectively. Some numerical instability was found for point mass located near the clamped end, probably because of very small eigenfrequency variations between unperturbed and perturbed configuration.

\subsection{Stability of identification to errors on the frequency data}

In order to test the robustness of the method, the identification for the cantilever in bending vibration was carried out by perturbing the noise-free eigenvalues $\widetilde{\lambda}_{n}, n=1,2$ as follows

$$
\sqrt{\tilde{\lambda}_{n}^{e r r}}=\sqrt{\widetilde{\lambda}_{n}}+\tau_{n}
$$

Here, $\tau_{n}$ is a random Gaussian variable with vanishing mean and standard

390 deviation $\sigma$ such that $3 \sigma=\Pi\left(\sqrt{\lambda_{n}}-\sqrt{\tilde{\lambda}_{n}}\right)$, where $\Pi$ is the maximum admitted error. Ten thousands simulations were performed by fixing the mass position at $s=0.55 \mathrm{~L}$, and investigating several levels of the maximum error $\Pi$ and of the normalized mass intensiy $M /(\rho L)$. Table 2 collects the results obtained for the set of values $\Pi=0.05,0.10,0.15,0.20$ and $M /(\rho L)=$ 
ble and accurate, with percentage deviations for $\Pi=0.20$, defined as the ratio between the standard deviation and the average of considered parameter, less than $2 \%$ and $5 \%$ for the mass position and the mass intensity, respectively.

\section{Conclusions}

Nanobeams are commonly used for mass-sensing, provided by the shift of natural frequencies from attached nanoparticles. At the scale of these sensors, the hypotheses of the classical continuum mechanics are no longer valid due to the discreteness of the matter. Thus generalized continuum mechanics theories should be used to capture the dynamic features of the system. To the authors on developing a method for the identification of the mass from minimal eigenfrequency data. The first order approximation for the eigenvalues of the beam with attached mass have been obtained for supported and cantilever boundary conditions, which seems to be in good agreement with the exact results for identification of the mass intensity by a pair of natural frequencies; the first two have been considered in the numerical calculations presented in this work. Likewise the mass position can be uniquely identified, up to a symmetrical point in the supported case, and up to critical ratio of the two frequency shifts in the the position, and somewhat worse for the intensity. Moreover, the effect of the frequency measurement errors on the estimated variables (mass intensity and 
location) has been illustrated with a statistical analysis, showing the robustness of the identification method. The results obtained herein encourage the use of bending vibration of nanobeams as a sensing technique, and show the feasibility of using strain gradient theories -more suitable for the analysis of solids at the nanoscale- for the identification of mass changes. Furthermore, the present study opens the possibility to investigate the identification of a point mass of finite - not necessarily small - magnitude. To that aim, the methods presented ${ }_{430}$ in [9] and [10] may be useful.

\section{Acknowledgements}

The authors from Universidad Carlos III de Madrid wish to acknowledge Ministerio de Economía y Competitividad de España for the financial support, under Grant number DPI2014-57989-P. The authors from University of Udine gratefully acknowledge the financial support of the National Research Project PRIN 2015TT JN95 'Identification and monitoring of complex structural systems'.

\section{References}

[1] K. Eom, H. S. Park, D. S. Yoon, T. Kwon, Nanomechanical resonators and their applications in biological/chemical detection: Nanomechanics principles, Physics Reports 503 (2011) 115-163.

[2] Q. Wang, B. Arash, A review on applications of carbon nanotubes and graphenes as nano-resonator sensors, Computational Materials Science 82 (2014) 350-360.

${ }_{445}[3]$ B. Arash, J. W. Jiang, T. Rabczuk, A review on nanomechanical resonators and their applications in sensors and molecular transportation, Applied Physics Reviews 2 (2015) 021301. 
[4] I. Voiculescu, M. Zaghloul (Eds.), Nanocantilever Beams: Modeling, Fabrication, and Applications, CRC Press, 2015.

450

455

[14] R. D. Mindlin, H. F. Tiersten, Effects of couple-stresses in linear elasticity, Archive for Rational Mechanics and Analysis 11 (5) (1962) 415-448. 
[15] R. A. Toupin, Theories of elasticity with couple-stress, Archive for Rational Mechanics and Analysis 17 (2) (1964) 85-112.

[16] R. D. Mindlin, Micro-structure in linear elasticity, Archive for Rational Mechanics and Analysis 16 (1964) 51-78.

[17] R. D. Mindlin, Second gradient of strain and surface-tension in linear elasticity, International Journal of Solids and Structures 1 (1965) 417-438.

[18] F. Yang, A. C. M. Chong, D. C. C. Lam, P. Tong, Couple stress based strain gradient theory for elasticity, International Journal of Solids and Structures 39 (2002) 2731-2743.

[19] D. C. C. Lam, F. Yang, A. C. M. Chong, J. Wang, P. Tong, Experiments and theory in strain gradient elasticity, Journal of the Mechanics and Physics of Solids 51 (2003) 1477-1508.

[20] E. Kröner, Elasticity theory of materials with long range cohesive forces, International Journal of Solids and Structures 3 (1967) 731-742.

[21] J. Krumhansl, Some considerations of the relation between solid state physics and generalized continuum mechanics, in: E. Kröner (Ed.), Mechanics of Generalized Continua. IUTAM Symposia. Springer Berlin Heidelberg, 1968, pp. 298-311.

[22] I. A. Kunin, The theory of elastic media with microstructure and the theory of dislocations, in: E. Kröner (Ed.), Mechanics of Generalized Continua. IUTAM Symposia. Springer Berlin Heidelberg, 1968, pp. 321-329.

[23] A. C. Eringen, Nonlocal polar elastic continua, International Journal of Engineering Science 10 (1) (1972) 1-16.

[24] A. C. Eringen, Linear theory of nonlocal elasticity and dispersion of planewaves, International Journal of Engineering Science 10 (5) (1972) 233-248. 
[25] A. C. Eringen, D. G. B. Edelen, Nonlocal elasticity, International Journal of Engineering Science 10 (3) (1972) 233-248.

[26] A. C. Eringen, On differential-equations of nonlocal elasticity and solutions of screw dislocation and surface-waves, Journal of Applied Physics 54 (9) (1983) 4703-4710.

[27] J. Peddieson, G. R. Buchanan, R. P. McNitt, Application of nonlocal continuum models to nanotechnology, International Journal of Engineering Science 41 (3-5) (2003) 305-312.

[28] M. A. Eltaher, M. E. Khater, S. A. Emam, A review on nonlocal elastic models for bending, buckling, vibrations, and wave propagation of nanoscale beams, Applied Mathematical Modelling 40 (2016) 4109-4128.

[29] H. Rafii-Tabar, E. Ghavanloo, S. A. Fazelzadeh, Nonlocal continuum-based modeling of mechanical characteristics of nanoscopic structures, Physics Reports 638 (2016) 1-97.

[30] H. T. Thai, T. P. Vo, T. K. Nguyen, S. E. Kim, A review of continuum mechanics models for size-dependent analysis of beams and plates, Composite Structures 177 (2017) 196-219.

[31] M. A. Eltaher, M. A. Agwa, F. F. Mahmoud, Nanobeam sensor for measuring a zeptogram mass, International Journal of Mechanics and Materials in Design 12 (2016) 211-221.

[32] T. Murmu, S. Adhikari, Nonlocal frequency analysis of nanoscale biosensors, Sensors and Actuators A: Physical 173 (2012) 41-48.

[33] X. F. Li, G. J. Tang, Z. B. Shen, K. Lee, Resonance frequency and mass identification of zeptogram-scale nanosensor based on the nonlocal beam theory, Ultrasonics 55 (2015) 75-84. 
[34] M. Zarepour, S. A. Hosseini, M. Ghadiri, Free vibration investigation of nano mass sensor using differential transformation method, Applied Physics A 123 (2017) 181.

[35] G. Romano, R. Barretta, M. Diaco, F. Marotti de Sciarra, Constitutive boundary conditions and paradoxes in nonlocal elastic nanobeams, International Journal of Mechanical Sciences 121 (2017) 151-156.

[36] E. Benvenuti, A. Simone, One-dimensional nonlocal and gradient elasticity: Closed-form solution and size effect, Mechanics Research Communications 48 (2013) 46-51.

[37] Q. Wang, K. M. Liew, Application of nonlocal continuum mechanics to static analysis of micro- and nano-structures, Physics Letters A 363 (3) (2007) 236-242.

${ }_{535}$ [38] N. Challamel, C. M. Wang, The small length scale effect for a non-local cantilever beam: a paradox solved, Nanotechnology 19 (2008) 345703(7).

[39] C. Wang, S. Kitipornchai, C. Lim, M. Eisenberger, Beam bending solutions based on nonlocal Timoshenko beam theory, Journal of Engineering Mechanics 134 (6) (2008) 475-481.

${ }_{540}$ [40] N. Challamel, Z. Zhang, C. M. Wang, J. N. Reddy, Q. Wang, T. Michelitsch, B. Collet, On nonconservativeness of Eringen's nonlocal elasticity in beam mechanics: correction from a discrete-based approach, Archive of Applied Mechanics 84 (2014) $1275-1292$.

[41] P. Lu, H. P. Lee, C. Lu, P. Q. Zhang, Dynamic properties of flexural beams using a nonlocal elasticity model, Journal of Applied Physics 99 (2006) 1 9.

[42] A. C. Eringen, Theory of nonlocal elasticity and some applications, Res Mechanica 21 (4) (1987) 313-342. 
[43] P. Khodabakhshia, J. N. Reddy, A unified integro-differential nonlocal model, International Journal of Engineering Science 95 (2015) 60-75.

[44] Y. B. Wang, X. W. Zhu, H. H. Dai, Exact solutions for the static bending of Euler-Bernoulli beams using Eringen's two-phase local/nonlocal model, AIP Advances 6 (2016) 085114.

[45] X. Zhu, Y. Wang, H. H. Dai, Buckling analysis of Euler-Bernoulli beams using Eringen's two-phase nonlocal model, International Journal of Engineering Science 1116 (2017) 130-140.

[46] K. Eptaimeros, C. C. Koutsoumaris, G. Tsamasphyros, Nonlocal integral approach to the dynamical response of nanobeams, International Journal of Mechanical Science 115-116 (2016) 68-80.

[47] J. Fernández-Sáez, R. Zaera, Vibrations of Bernoulli-Euler beams using the two-phase nonlocal elasticity theory, International Journal of Engineering Science 119 (2017) 232-248.

[48] N. A. Fleck, J. W. Hutchinson, Strain gradient plasticity, Advances in Applied Mechanics 33 (1997) 295-361.

[49] M. H. Kahrobaiyan, M. Asghari, M. T. Ahmadian, Strain gradient beam element, Finite Elements in Analysis and Design 68 (2013) 63-75.

[50] S. Kong, S. Zhou, Z. Nie, K. Wang, Static and dynamic analysis of microbeams based on strain gradient elasticity theory, International Journal of Engineering Science 47 (2009) 487-498.

570 [51] B. Wang, J. Zhao, S. Zhou, A microscale Timoshenko beam model based on strain gradient elasticity theory, European Journal of Mechanics A-Solids 29 (2010) 837-843. 
[52] B. Akgoz, O. Civalek, Longitudinal vibration analysis of strain gradient bars made of functionally graded materials(FGM), Composites: Part B 55 (2013) 263-268.

[53] B. Akgoz, O. Civalek, Longitudinal vibration analysis for microbars based on strain gradient elasticity theory, Journal of Vibration and Control 20 (2014) 606-616.

[54] B. Akgoz, O. Civalek, Bending analysis of embedded carbon nanotubes resting on an elastic foundation using strain gradient theory, Acta Astronautica 119 (2016) 1-12.

[55] H. Mohammadi, M. Mahzoon, Thermal effects on postbuckling of nonlinear microbeams based on the modified strain gradient theory, Composite Structures 106 (2013) 764-776.

[56] E. M. Miandoab, A. Yousefi-Koma, H. N. Pishkenari, Nonlocal and strain gradient based model for electrostatically actuated silicon nano-beams, Microsystem Technologies 21 (2015) 457-464.

[57] B. Zhang, Y. He, D. Liu, Z. Gan, L. Shen, Non-classical timoshenko beam element based on the strain gradient elasticity theory, Finite Elements in Analysis and Design 79 (2014) 22-39.

[58] A. Morassi, J. Fernández-Sáez, R. Zaera, J. A. Loya, Resonator-based detection in nanorods, Mechanical Systems and Signal Processing 93 (2017) 645-660.

[59] B. Akgoz, O. Civalek, Strain gradient elasticity and modified couple stress models for buckling analysis of axially loaded micro-scaled beams, International Journal of Engineering Science 49 (2011) 1268-1280.

[60] D. Kumar, C. Heinrich, A. M. Waas, Buckling analysis of carbon nanotubes 
modeled using nonlocal continuum theories, Journal of Applied Physics 103 (2008) 073521.

[61] O. Civalek, C. Demir, A simple mathematical model of microtubules surrounded by an elastic matrix by nonlocal finite element method, Applied Mathematics and Computation 289 (2016) 335-352.

[62] R. Courant, D. Hilbert, Methods of Mathematical Physics, (first English edition), Interscience Publishers Inc., New York, 1966.

[64] L. Rubio, J. Fernández-Sáez, A. Morassi, Identification of two cracks with different severity in beams and rods from minimal frequency data, Journal of Vibration and Control 22 (2016) 3102-3117. 


\section{Table Captions}

${ }_{610}$ Table 1. Geometrical properties of the nanobeam. Length in $\mu m$.

Table 2. Results of identification for the cantilever nanobeam with noise data as in (64) for point mass $M$ located at $s=0.55 L$, for increasing (normalized) mass intensity $M / \rho L$ and different values of the maximum error П. Percentage errors: $\operatorname{err}(s)=100 \times\left(s_{\text {average }}-s_{\text {exact }}\right) / L, \operatorname{err}(M /(\rho L))=100 \times\left(M_{\text {average }}-\right.$ $\left.{ }_{615} M_{\text {exact }}\right) / M_{\text {exact }}$. 


\section{Figure Captions}

Figure 1. Typical behaviour of the function $g(s)$ defined in equation (54).

Figure 2. Simply supported nanobeam. Normalized first eigenvalue versus dimensionless point-mass, for different mass position and different values of the

${ }_{620}$ length scale parameter.

Figure 3. Simply supported nanobeam. Normalized second eigenvalue versus dimensionless point-mass, for different mass position and different values of the length scale parameter

Figure 4. Cantilever nanobeam. Normalized first eigenvalue versus dimension${ }_{625}$ less point-mass, for different mass position and different values of the length scale parameter.

Figure 5. Cantilever nanobeam. Normalized second eigenvalue versus dimensionless point-mass, for different mass position and different values of the length scale parameter.

Figure 6. Supported nanobeam: identification using the variations of the first two eigenfrequencies of the bending vibration for different values of the point-mass. Left column: percentage errors on the mass position, $\operatorname{err}(s)=$ $100 \times\left(s_{\text {ident }}-s_{\text {exact }}\right) / L$. Right column: percentage errors on the mass intensity, $\operatorname{err}(M /(\rho L))=100 \times\left(M_{\text {ident }}-M_{\text {exact }}\right) / M_{\text {exact }}$.

Figure 7. Cantilever nanobeam: identification using the variations of the first two eigenfrequencies of the bending vibration for different values of the point-mass. Left column: percentage errors on the mass position, $\operatorname{err}(s)=$ $100 \times\left(s_{\text {ident }}-s_{\text {exact }}\right) / L$. Right column: percentage errors on the mass intensity, $\operatorname{err}(M /(\rho L))=100 \times\left(M_{\text {ident }}-M_{\text {exact }}\right) / M_{\text {exact }}$. 
Table 1: Geometrical properties of the nanobeam. Length in $\mu \mathrm{m}$.

\begin{tabular}{ccc}
$\begin{array}{c}\text { Thickness } \\
h\end{array}$ & Width & Length \\
$b=2 h$ & $L=20 h$ \\
\hline 50 & 100 & 1000 \\
\hline
\end{tabular}


Table 2: Results of identification for the cantilever nanobeam with noise data as in (64)

for point mass $M$ located at $s=0.55 L$, for increasing (normalized) mass intensity $M / \rho L$ and different values of the maximum error $\Pi$. Percentage errors: $\operatorname{err}(s)=100 \times\left(s_{\text {average }}-\right.$ $\left.s_{\text {exact }}\right) / L, \operatorname{err}(M /(\rho L))=100 \times\left(M_{\text {average }}-M_{\text {exact }}\right) / M_{\text {exact }}$.

\begin{tabular}{|c|c|c|c|c|c|c|c|c|c|}
\hline \multirow{2}{*}{$\begin{array}{l}\text { Case } \\
M / \rho L\end{array}$} & \multirow[t]{2}{*}{ Stat. property } & \multicolumn{4}{|c|}{ Mass position $s / L$} & \multicolumn{4}{|c|}{ Mass intensity $M / \rho L$} \\
\hline & & $\Pi=0.05$ & $\Pi=0.10$ & $\Pi=0.15$ & $\Pi=0.20$ & $\Pi=0.05$ & $\Pi=0.10$ & $\Pi=0.15$ & $\Pi=0.20$ \\
\hline \multirow[t]{5}{*}{0.010} & $\min$ & 0.541 & 0.534 & 0.520 & 0.513 & 0.0093 & 0.0089 & 0.0086 & 0.0079 \\
\hline & $\max$ & 0.562 & 0.567 & 0.584 & 0.589 & 0.0103 & 0.0109 & 0.0111 & 0.0117 \\
\hline & average & 0.552 & 0.552 & 0.552 & 0.552 & 0.0098 & 0.0098 & 0.0098 & 0.0098 \\
\hline & error $(\%)$ & $0.2 \%$ & $0.2 \%$ & $0.2 \%$ & $0.2 \%$ & $-1.7 \%$ & $-1.7 \%$ & $-1.6 \%$ & $-1.6 \%$ \\
\hline & std dev & 0.0024 & 0.0048 & 0.0073 & 0.0097 & 0.00012 & 0.00024 & 0.00036 & 0.00048 \\
\hline \multirow[t]{5}{*}{0.025} & $\min$ & 0.544 & 0.537 & 0.524 & 0.516 & 0.0229 & 0.0218 & 0.0203 & 0.0197 \\
\hline & $\max$ & 0.563 & 0.572 & 0.578 & 0.587 & 0.0252 & 0.0266 & 0.0275 & 0.0282 \\
\hline & average & 0.554 & 0.554 & 0.554 & 0.554 & 0.0240 & 0.0240 & 0.0240 & 0.0240 \\
\hline & error $(\%)$ & $0.4 \%$ & $0.4 \%$ & $0.4 \%$ & $0.4 \%$ & $-4.1 \%$ & $-4.1 \%$ & $-4.0 \%$ & $-4.1 \%$ \\
\hline & std dev & 0.0023 & 0.0048 & 0.0071 & 0.0096 & 0.00029 & 0.00058 & 0.00087 & 0.00115 \\
\hline \multirow[t]{5}{*}{0.050} & $\min$ & 0.550 & 0.541 & 0.529 & 0.520 & 0.0441 & 0.0425 & 0.0390 & 0.0364 \\
\hline & $\max$ & 0.567 & 0.577 & 0.584 & 0.593 & 0.0481 & 0.0509 & 0.0524 & 0.0548 \\
\hline & average & 0.559 & 0.559 & 0.558 & 0.559 & 0.0461 & 0.0461 & 0.0461 & 0.0461 \\
\hline & error $(\%)$ & $0.9 \%$ & $0.9 \%$ & $0.8 \%$ & $0.9 \%$ & $-7.8 \%$ & $-7.8 \%$ & $-7.8 \%$ & $-7.8 \%$ \\
\hline & std dev & 0.0023 & 0.0046 & 0.0069 & 0.0092 & 0.00055 & 0.00109 & 0.00164 & 0.00218 \\
\hline \multirow[t]{5}{*}{0.100} & $\min$ & 0.558 & 0.549 & 0.541 & 0.522 & 0.0818 & 0.0780 & 0.0737 & 0.0721 \\
\hline & $\max$ & 0.573 & 0.584 & 0.592 & 0.601 & 0.0895 & 0.0936 & 0.0972 & 0.1035 \\
\hline & average & 0.566 & 0.566 & 0.566 & 0.566 & 0.0858 & 0.0858 & 0.0858 & 0.0859 \\
\hline & error $(\%)$ & $1.6 \%$ & $1.6 \%$ & $1.6 \%$ & $1.6 \%$ & $-14.2 \%$ & $-14.2 \%$ & $-14.2 \%$ & $-14.1 \%$ \\
\hline & std dev & 0.0022 & 0.0044 & 0.0067 & 0.0088 & 0.00099 & 0.00201 & 0.00300 & 0.00397 \\
\hline \multirow[t]{5}{*}{0.150} & $\min$ & 0.563 & 0.553 & 0.543 & 0.539 & 0.1156 & 0.1110 & 0.1046 & 0.0989 \\
\hline & $\max$ & 0.581 & 0.588 & 0.595 & 0.604 & 0.1261 & 0.1311 & 0.1383 & 0.1419 \\
\hline & average & 0.572 & 0.572 & 0.572 & 0.572 & 0.1206 & 0.1206 & 0.1206 & 0.1206 \\
\hline & error $(\%)$ & $2.2 \%$ & $2.2 \%$ & $2.2 \%$ & $2.2 \%$ & $-19.6 \%$ & $-19.6 \%$ & $-19.6 \%$ & $-19.6 \%$ \\
\hline & std dev & 0.0021 & 0.0043 & 0.0064 & 0.0085 & 0.00137 & 0.00272 & 0.00409 & 0.00543 \\
\hline \multirow[t]{5}{*}{0.200} & $\min$ & 0.570 & 0.561 & 0.553 & 0.546 & 0.1456 & 0.1396 & 0.1290 & 0.1272 \\
\hline & $\max$ & 0.585 & 0.591 & 0.603 & 0.605 & 0.1578 & 0.1631 & 0.1714 & 0.1821 \\
\hline & average & 0.578 & 0.578 & 0.578 & 0.578 & 0.1517 & 0.1517 & 0.1517 & 0.1516 \\
\hline & error $(\%)$ & $2.8 \%$ & $2.8 \%$ & $2.8 \%$ & $2.8 \%$ & $-24.2 \%$ & $-24.2 \%$ & $-24.2 \%$ & $-24.2 \%$ \\
\hline & std dev & 0.0020 & 0.0041 & 0.0062 & 0.0082 & 0.00170 & 0.00335 & 0.00502 & 0.00677 \\
\hline
\end{tabular}




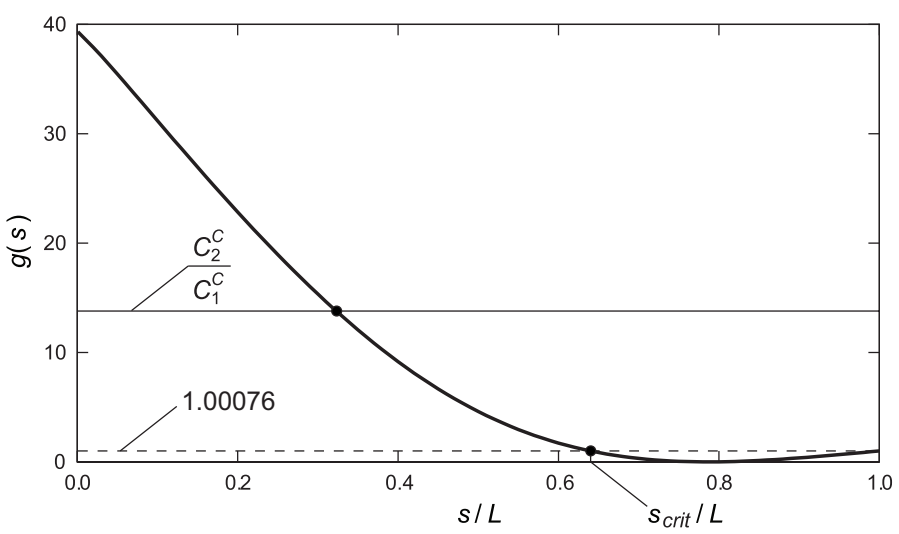

Figure 1: Typical behaviour of the function $g(s)$ defined in equation (54). 
(a) $h / l=10$

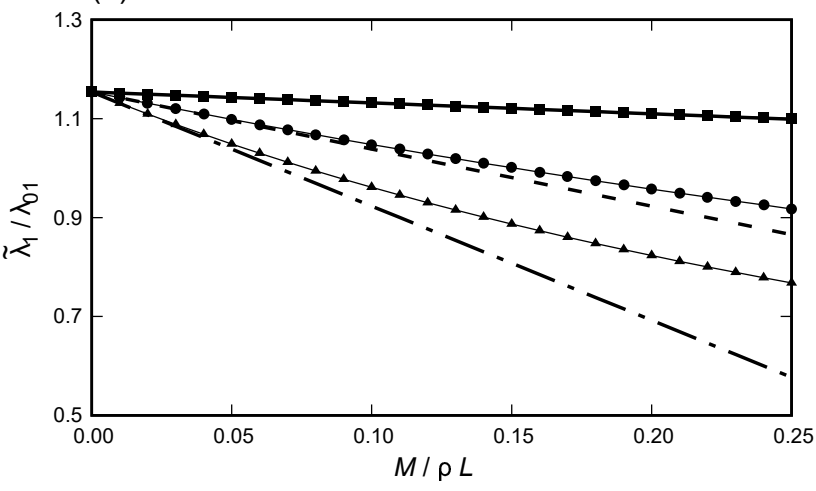

(c) $h / l=2$

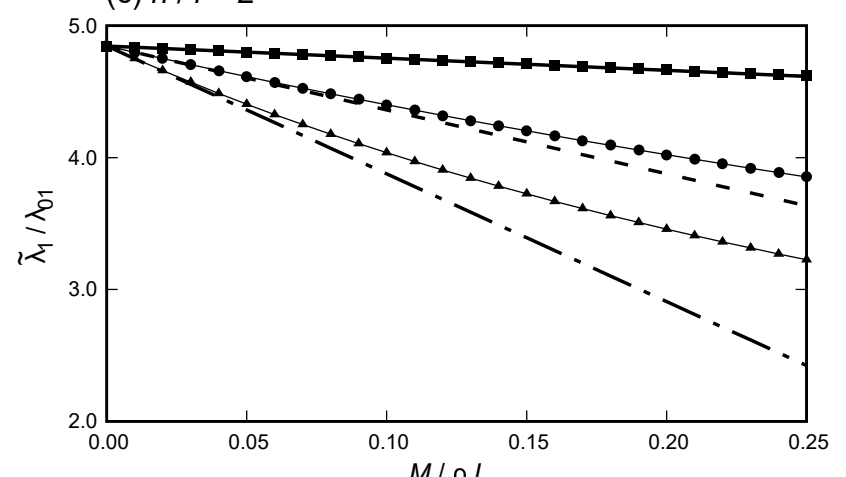

(b) $h / l=5$

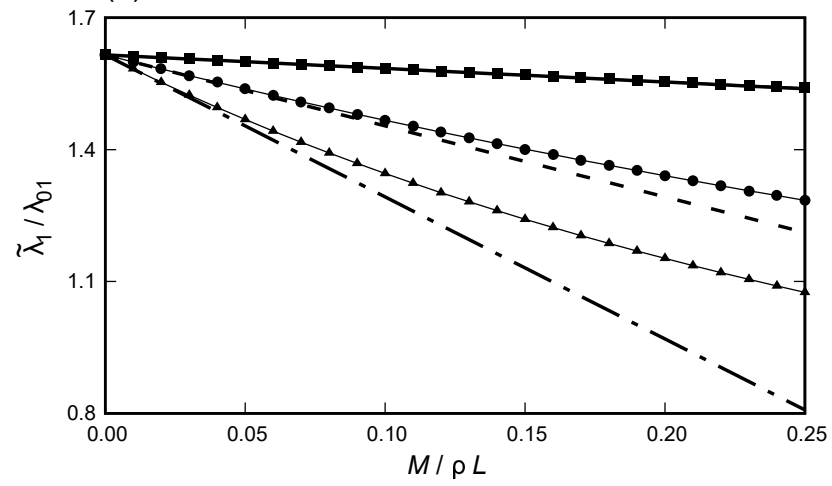

(d) $h / l=1$

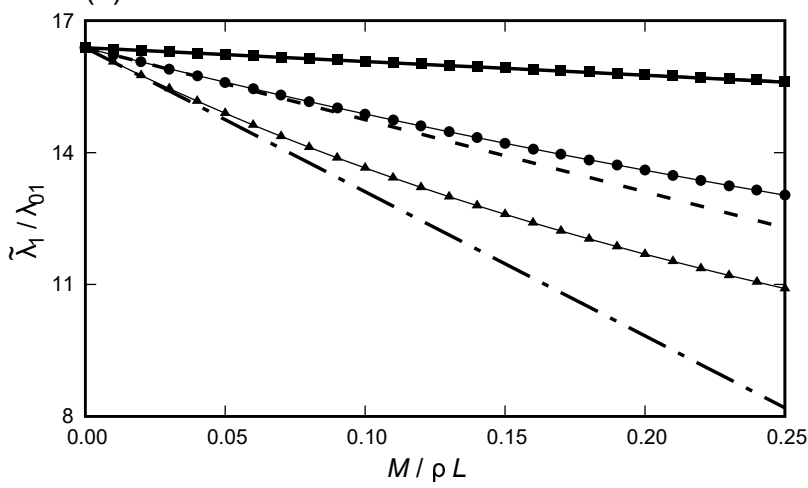

— $s=0.10 L$ Exact $-\longrightarrow s=0.10 \angle$ Perturb. $\quad \longrightarrow s=0.25 L$ Exact

- $\quad s=0.25 L$ Perturb. $\quad \longrightarrow s=0.50 L$ Exact $\quad-s=0.50 L$ Perturb.

Figure 2: Simply supported nanobeam. Normalized first eigenvalue versus dimensionless point-mass, for different mass position and different values of the length scale parameter. 
(a) $h / I=10$

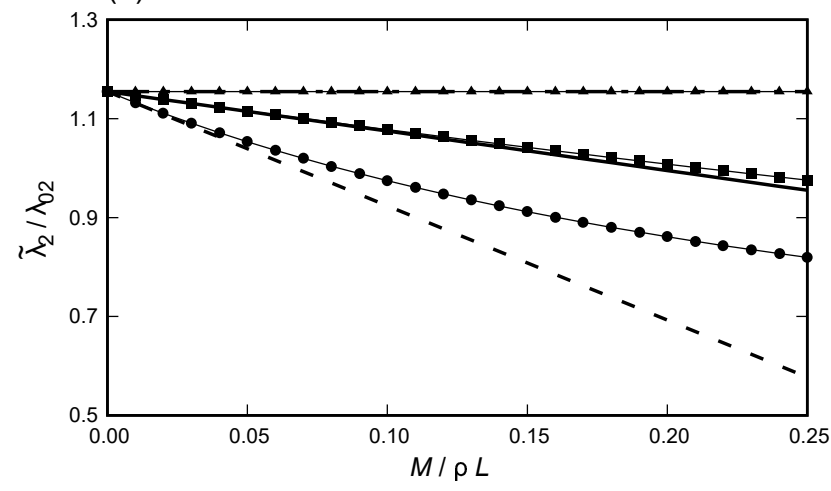

(c) $h / l=2$

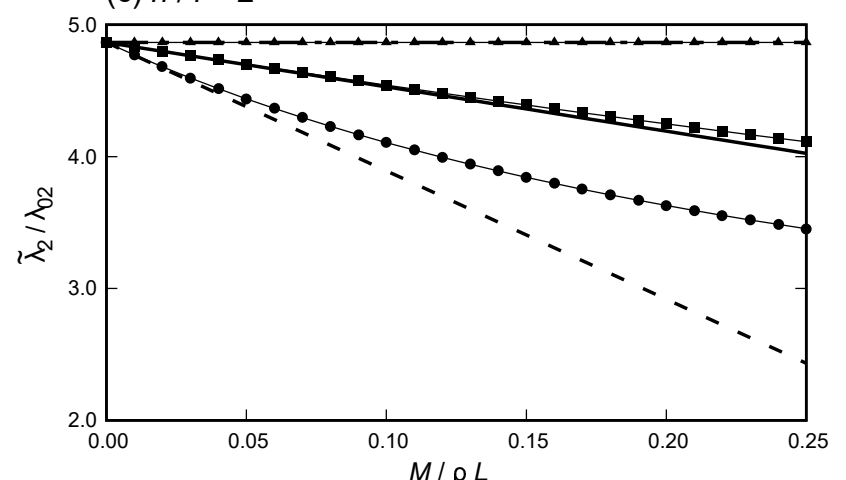

(b) $h / l=5$

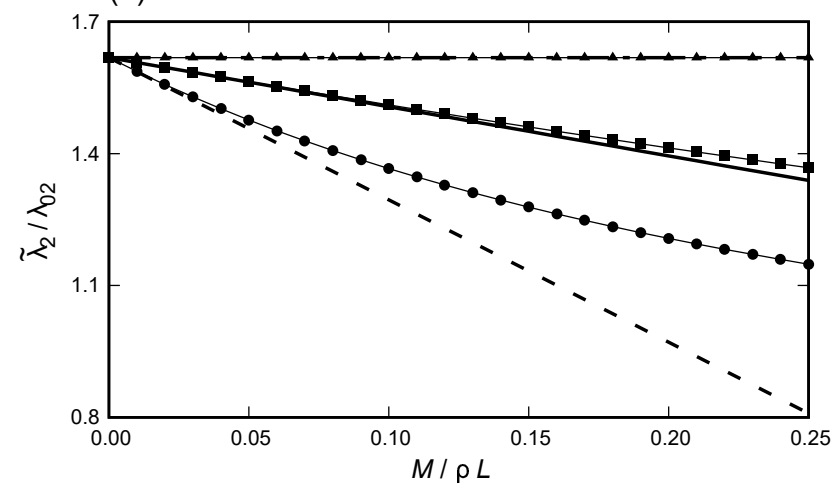

(d) $h / l=1$

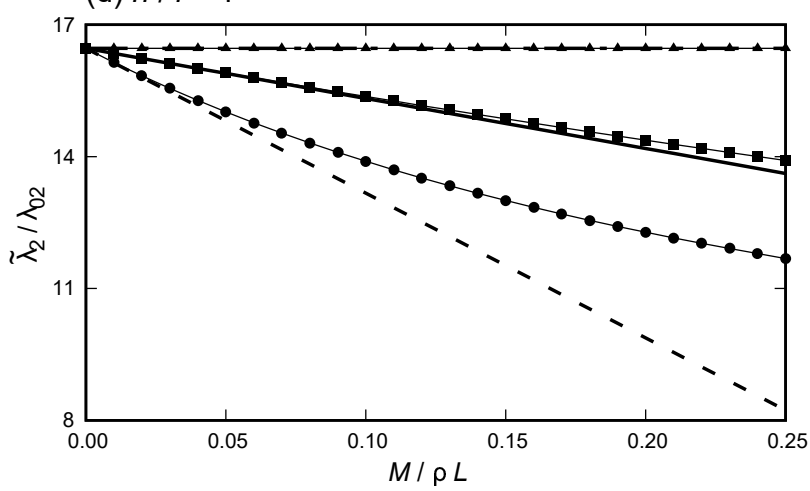

$\longrightarrow-s=0.10 \angle$ Exact $\longrightarrow s=0.10 \angle$ Perturb. $\longrightarrow s=0.25 L$ Exact

- $\quad s=0.25 L$ Perturb. $\quad \longrightarrow s=0.50 L$ Exact $\quad-s=0.50 L$ Perturb.

Figure 3: Simply supported nanobeam. Normalized second eigenvalue versus dimensionless point-mass, for different mass position and different values of the length scale parameter. 
(a) $h / /=10$

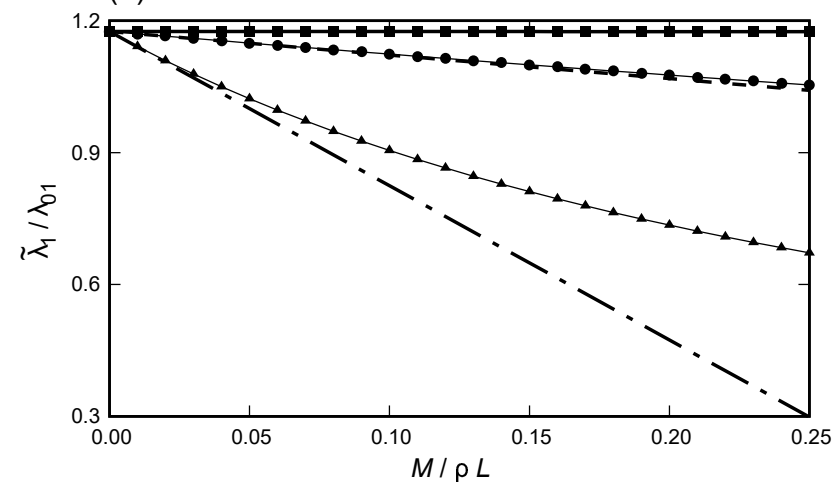

(c) $h / I=2$

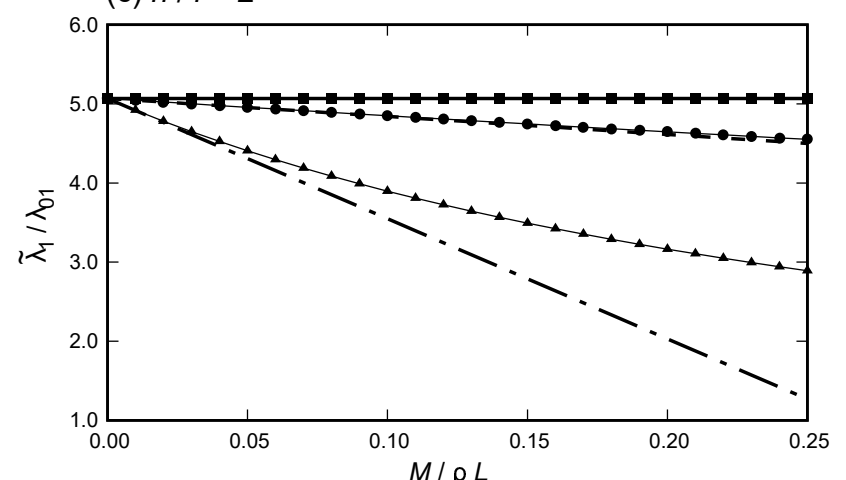

(b) $h / l=5$

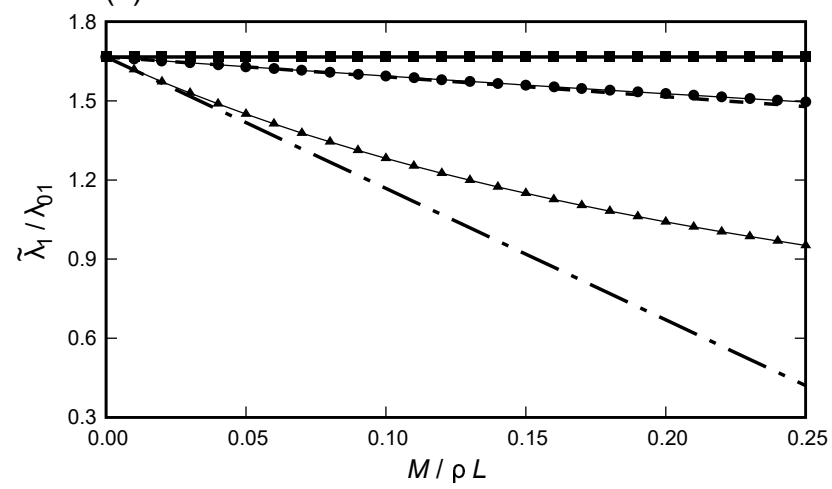

(d) $h / l=1$

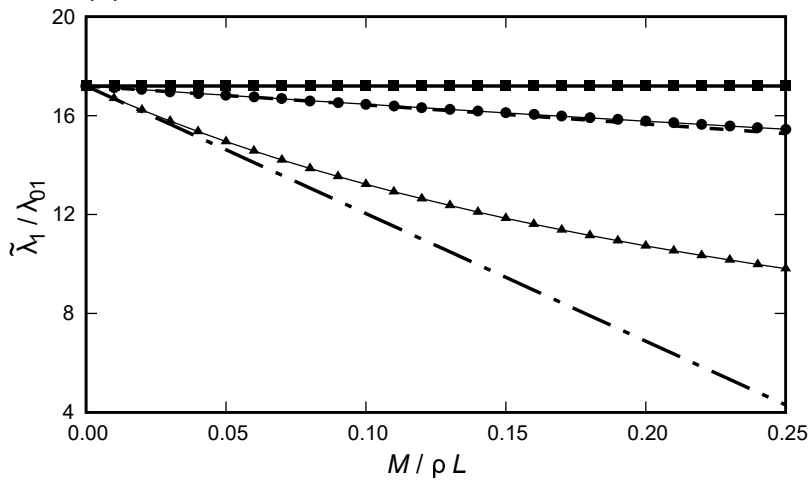

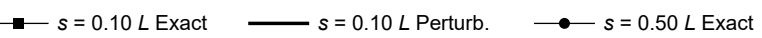

- $\quad s=0.50 L$ Perturb. $\_s=0.90 L$ Exact $\longrightarrow-s=0.90 L$ Perturb.

Figure 4: Cantilever nanobeam. Normalized first eigenvalue versus dimensionless point-mass, for different mass position and different values of the length scale parameter. 
(a) $h / /=10$

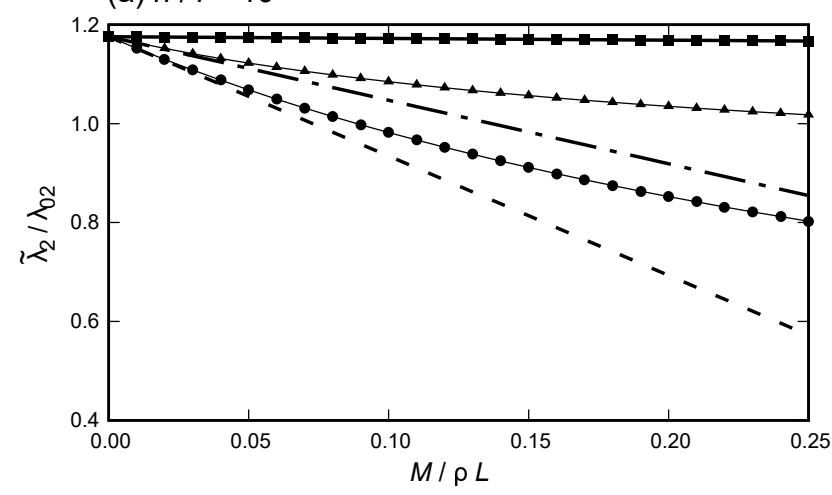

(c) $h / I=2$

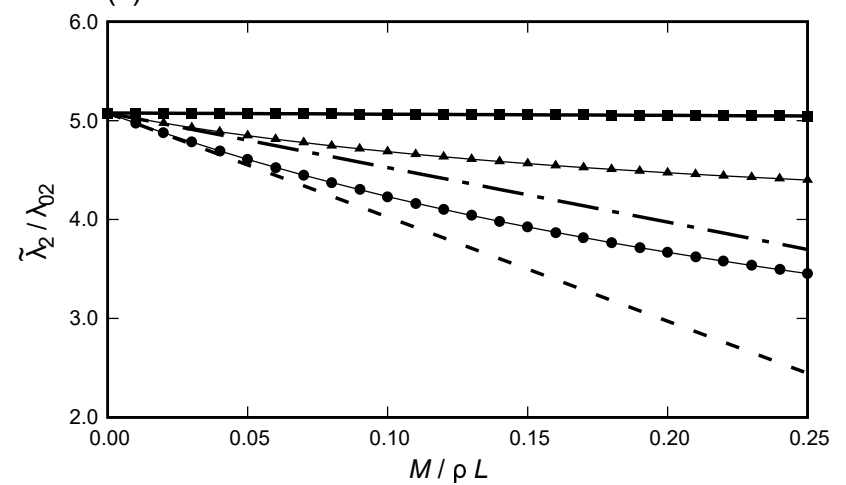

(b) $h / l=5$

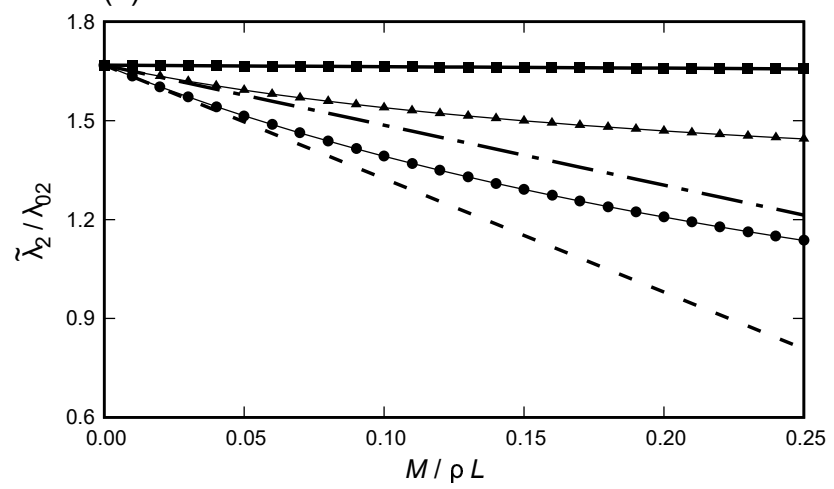

(d) $h / /=1$

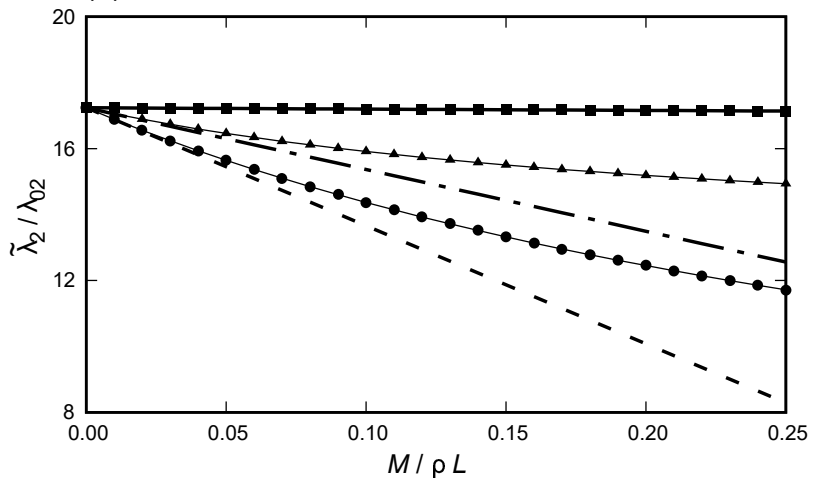

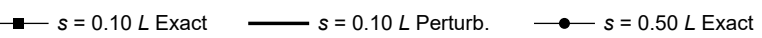

- - $s=0.50 L$ Perturb. $\quad \longrightarrow s=0.90 L$ Exact $\quad-s=0.90 L$ Perturb.

Figure 5: Cantilever nanobeam. Normalized second eigenvalue versus dimensionless pointmass, for different mass position and different values of the length scale parameter. 
(a)

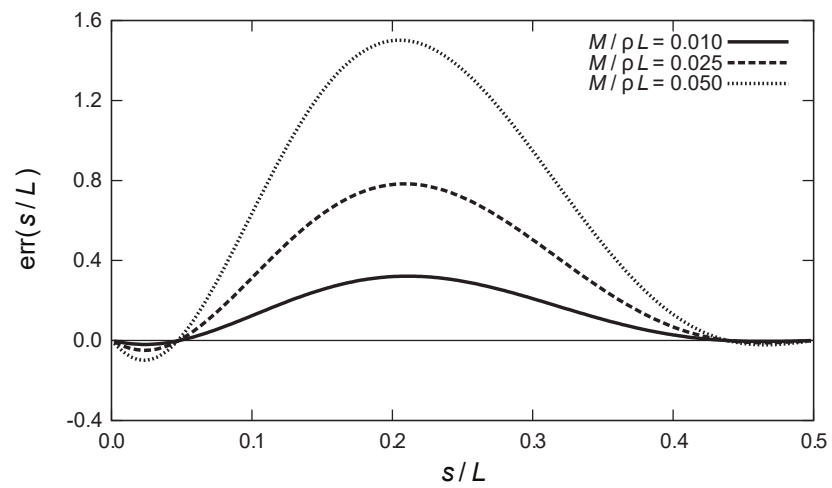

(c)

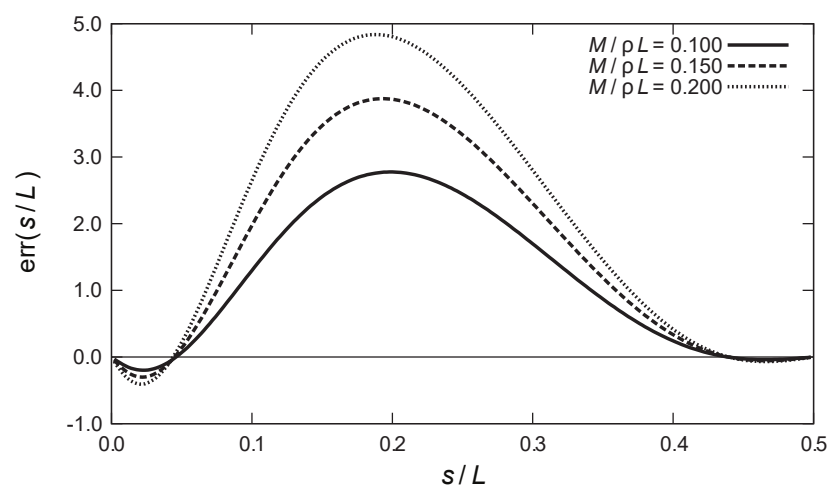

(b)

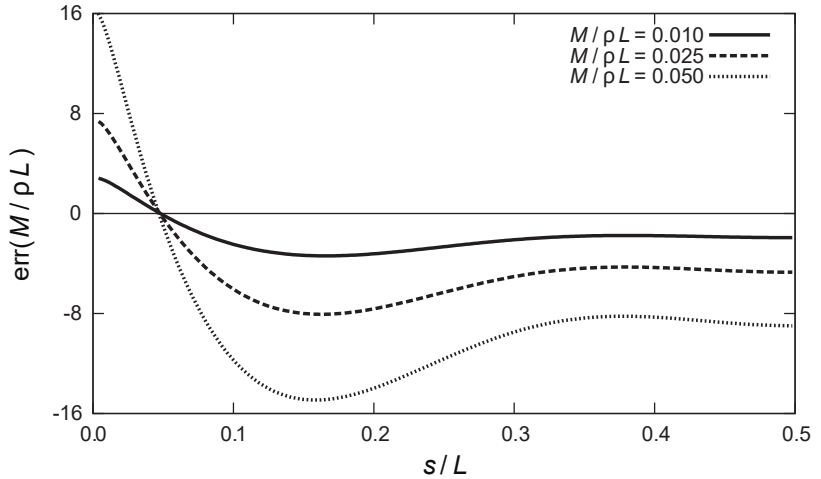

(d)

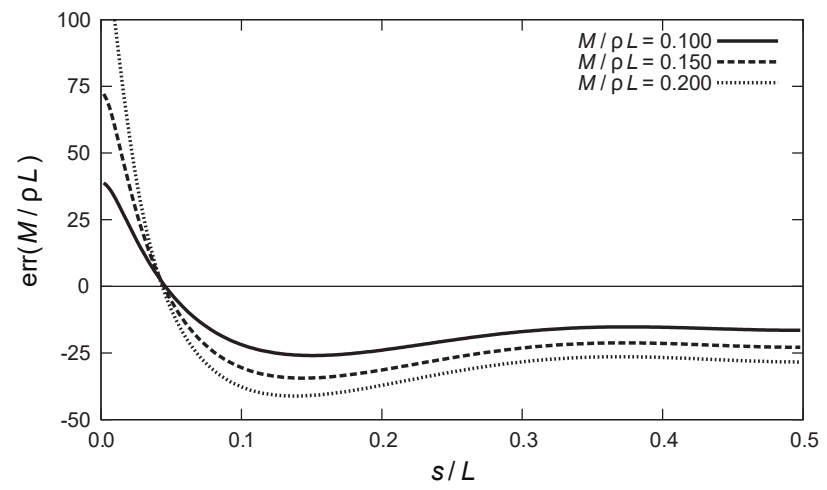

Figure 6: Supported nanobeam (19)-(29): identification using the variations of the first two eigenfrequencies of the bending vibration for different values of the point-mass. Left column: percentage errors on the mass position, $\operatorname{err}(s)=100 \times\left(s_{\text {ident }}-s_{\text {exact }}\right) / L$. Right column: percentage errors on the mass intensity, $\operatorname{err}(M /(\rho L))=100 \times\left(M_{\text {ident }}-M_{\text {exact }}\right) / M_{\text {exact }}$. 
(a)

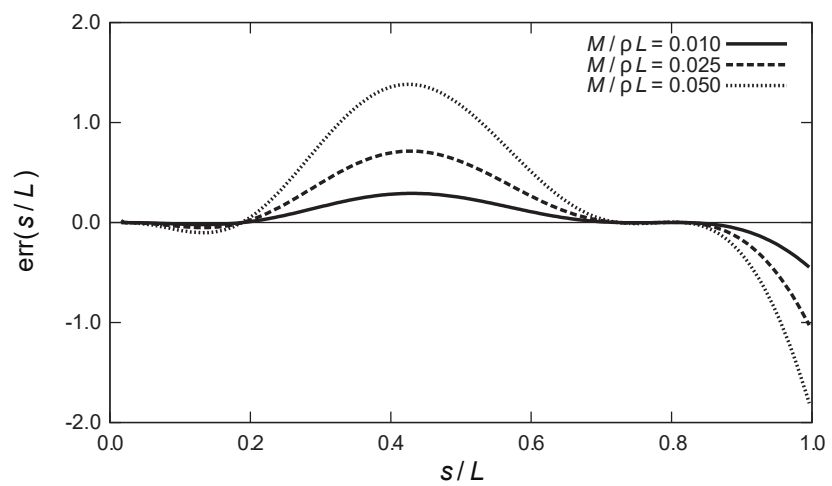

(c)

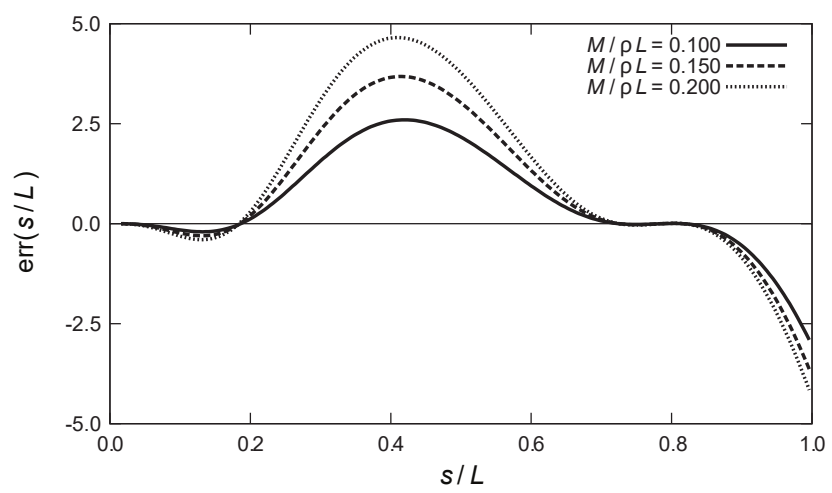

(b)

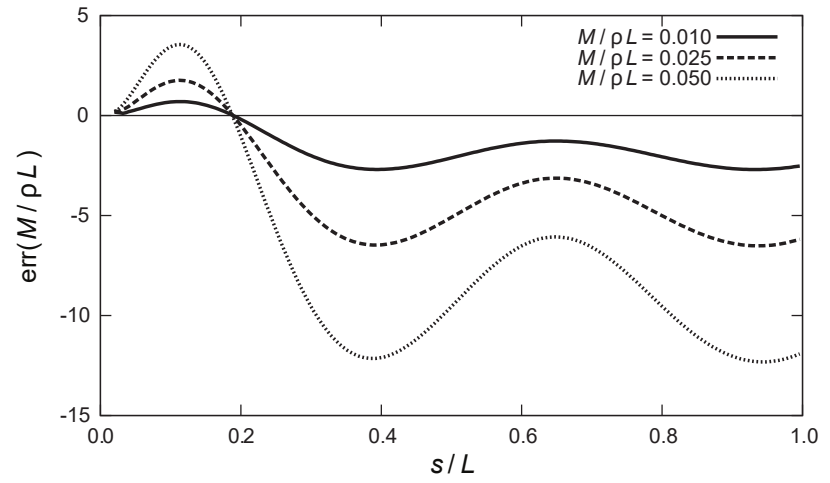

(d)

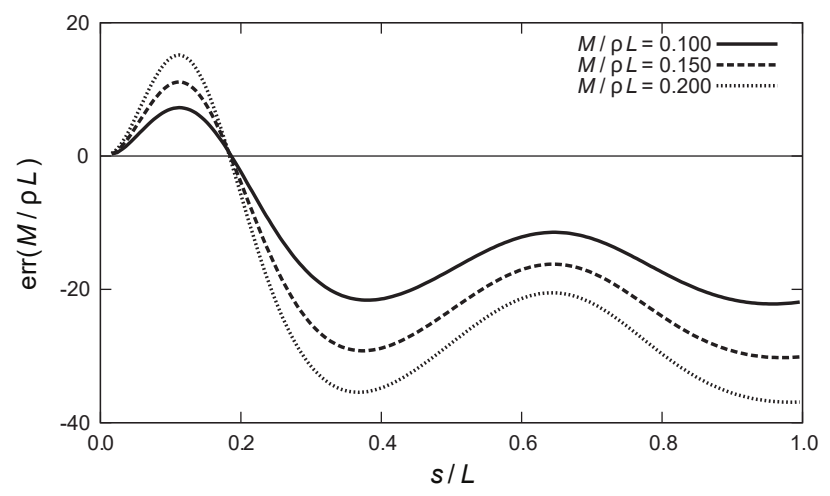

Figure 7: Cantilever nanobeam: identification using the variations of the first two eigenfrequencies of the bending vibration for different values of the point-mass. Left column: percentage errors on the mass position, $\operatorname{err}(s)=100 \times\left(s_{\text {ident }}-s_{\text {exact }}\right) / L$. Right column: percentage errors on the mass intensity, $\operatorname{err}(M /(\rho L))=100 \times\left(M_{\text {ident }}-M_{\text {exact }}\right) / M_{\text {exact }}$. 